\title{
Calidad en datos geográficos, geoservicios y productores de datos: análisis crítico
}

\author{
Francisco Javier Ariza* \\ Antonio F. Rodríguez ${ }^{* *}$
}

Recibido 02 de marzo de 2018, aceptado 29 de agosto de 2018

\section{Resumen}

En este trabajo se presenta un análisis, centrado en la calidad de datos y servicios geográficos, asumiendo que la aproximación habitual a la calidad de datos debe ser extendida a los servicios web dentro de la Arquitectura Orientada a Servicios que sirve de base a las IDE. Para tener un panorama más completo, también se considerará la calidad del productor de datos que implementa ambas clases de recursos. El análisis se realiza con un juicio crítico desde la perspectiva de las exigencias que los numerosos cambios y tendencias plantean a los tres elementos anteriores. Así, se analiza la situación actual en cuanto a estándares disponibles y prácticas habituales y se enjuician ciertas limitaciones de los estándares y métodos usuales desde la perspectiva anteriormente indicada. El trabajo ofrece líneas de mejora para superar esta situación, muchas de las cuales suponen abrir campos de investigación muy necesarios.

Palabras clave: calidad, interoperabilidad, calidad de recursos, modelo de calidad, aspectos de la calidad.

\section{Resumo}

Neste trabalho se apresenta uma análise, centrada na qualidade de dados e serviços geográficos, considerando-se que a aproximação habitual da qualidade de dados deve ser estendida aos serviços web dentro da Arquitetura Orientada a Serviços que serve de base às IDEs. Para se ter um panorama mais completo, também se considerará a qualidade do produtor de dados que implementa ambas as classes de recursos.

* Departamento de Ingeniería Cartográfica, Geodésica y Fotogrametría, Universidad de Jaén, Campus Las Lagunillas, s/n, 23071 Jaén, España, correo electrónico: fjariza@ujaen.es

** Centro Nacional de Información Geográfica (CNIG), Instituto Geográfico Nacional (IGN) de España, Calle General Ibañez de Ibero, 328003 Madrid, España, correo electrónico: afrodriguez@fomento.es 
A análise se realiza com um juízo crítico desde a perspectiva das exigências que as numerosas mudanças e tendências representam para os três elementos anteriores. Sendo assim, se analisa a situação atual quanto a padrões disponíveis e práticas habituais e se julgar certas limitações dos padrões e métodos usuais desde a perspectiva anteriormente indicada. $\mathrm{O}$ trabalho oferece linhas de melhora para superar esta situação, muitas das quais se supõe abrir campos de investigação muito necessários.

Palavras chave: qualidade, interoperabilidade, qualidade de recursos, modelo de qualidade, aspectos da qualidade.

\begin{abstract}
This paper presents an analysis, focused on quality of geographic data and services, assuming that the usual approach to data quality need to be extended to web services following the basic Services Oriented Architecture for SDIs. The quality of the organization implementing the two kind of resources is also considered to have a more complete picture. The analysis is carried out with a critical judgment from the perspective of the demands that the numerous changes and tendencies demand on the three previous elements. Thus, the current situation regarding available standards and usual practices is analyzed and certain limitations of the usual standards and methods are judged from the perspective indicated above. The work offers lines of improvement to overcome this situation, many of which involve opening much-needed research fields.
\end{abstract}

Key words: quality, interoperability, resources quality, quality model, quality aspects.

La felicidad de tu vida depende de la calidad de tus pensamientos. Marco Aurelio

Preocúpate por la calidad, mucha gente no está preparada para un entorno donde la excelencia es lo que se espera.

Steve Jobs

\title{
Introducción
}

Hoy en día, es necesario concebir la actividad productiva de datos geoespaciales, o Información Geográfica (IG), en íntima conexión con el campo de las Tecnologías de la Información y las Comunicaciones (TIC). Además, se debe tener una clara orientación a la producción de recursos, ya sean bienes o servicios y, más concretamente, a la producción de modelos virtuales sobre cualquier aspecto de la realidad que nos rodea, y siempre con el objetivo de ayudar en la toma de decisiones. Estos 
modelos se presentan en forma de productos de datos (p.ej. mapas o conjuntos de datos espaciales) y geoservicios (p.ej. ruteros en la web) que se utilizan en la toma de decisiones. A nivel macro son la base para el desarrollo de proyectos, planes y políticas nacionales, es decir, programación de grandes inversiones. Pero, también tienen repercusión a nivel micro, como es el quehacer de las empresas y particulares (p.ej. agricultura de precisión, manejo de vehículos no tripulados, programación de rutas y actividades de ocio, entre otros). Por tanto, se puede afirmar que generar datos espaciales es una tarea importante que tiene consecuencias reales sobre el medio y la sociedad (Oxera, 2013). Esta actividad afecta a individuos concretos en su vida, bienestar, derechos y obligaciones; por ello las administraciones encargadas de generarlos y gestionarlos deben procurar que alcancen los niveles de calidad que permitan conseguir los resultados esperados. Los datos espaciales y, en general, las tecnologías de la IG, son herramientas de la buena gobernanza y juegan un papel primordial para conseguir los objetivos de desarrollo sostenible de la Organización de las Naciones Unidas (ONU). Por todos estos motivos la calidad es un tema candente en las agendas de trabajo de las organizaciones internacionales del campo geomático (Karpik y Musikhin, 2016), apoyado por el Instituto Panamericano de Geografía e Historia (IPGH) por medio de diversos proyectos de asistencia técnica.

Al igual que otras muchas formas tradicionales de actividad (p.ej. la docencia, las relaciones sociales, las relaciones comerciales, la producción industrial y muchas otras) ha cambiado radicalmente en los últimos años y está cambiando en la actualidad. La producción de IG también se enfrenta a un nuevo marco de trabajo como consecuencia de la evolución de las TIC, la globalización y de los cambios sociales y productivos que conllevan. En esta línea, tal como muestra la Tabla 1, son numerosos los cambios radicales a los que asistimos que afectan a la producción y uso de IG, tal y como los hemos entendido hasta ahora. Estos cambios condicionan su futuro inmediato $y$, en particular, el de los aspectos relacionados con la calidad y, como un aspecto esencial de ella en el marco TIC, también de la interoperabilidad. En este nuevo marco aparecen multitud de nuevos usuarios (democratización del uso), pero también multitud de nuevos productores. Así se habla de democratización de la producción o Información Geográfica Voluntaria, IGV (Goodchild, 2007) y de nuevas figuras como los "prosuarios": productores y usuarios a un mismo tiempo. Como consecuencia, están apareciendo sistemas productivos fuertemente descentralizados, en los que los esfuerzos federativos y confederativos, la hibridación de sistemas de producción (Cinnamon, 2014), etc., junto a sus consecuencias, son todavía poco claros y presentan numerosos riesgos respecto a la calidad. Además, esta situación lleva a los organismos tradicionalmente responsables de la cartografía oficial a tener que afrontar nuevos retos de cara al futuro. 
En el campo industrial se habla de industria 4.0 y de revolución industrial (Oesterreich y Teuteberg, 2016), pero si bien la IG es predominantemente digital desde hace años y se encuentra estrechamente ligada a las TIC, también se requiere una cierta revolución con motivo de la nueva realidad que se está conformando. Así, según (Dans, 2010), la transformación digital de las organizaciones, ya sean públicas o privadas, es inevitable y las que no la pongan en práctica perderán oportunidades y competitividad rápidamente, hasta quedar obsoletas y verse obligadas a cerrar, en el caso de las privadas, o volverse residuos irrelevantes, en el caso de las públicas. Por ello, de una manera genérica, hablaremos de riesgo, para referirnos a todos aquellos factores clave que pueden ser decisivos, de cara al futuro, para las organizaciones no oficiales que producen IG, o que ofrecen geoservicios, y que lastran su evolución hasta impedir una transformación digital completa.

También hablaremos de determinación de la calidad, como actividad clave para prevenir esos riesgos y evitarlos siempre que sea posible o, al menos, reducirlos al mínimo.

Volviendo a la idea de la transformación digital de las organizaciones en el campo de la producción de IG, es éste un aspecto especialmente interesante en la actualidad. Por un lado, hay algunos procesos de producción cartográfica difícilmente automatizables, que hacen que la cartografía sea en parte un arte, como la generalización, la simbolización, la conflación, la captura de datos o el case de bordes. Sin embargo, estamos asistiendo a la prometedora emergencia del aprendizaje automático (machine learning y deep learnning), que ya soluciona de manera automática procesos en los que hasta ahora era imprescindible la intervención humana interactiva (véase Rodrigo, 2017), lo que permite augurar un futuro muy fructífero en esta línea.

\section{Tabla 1}

Resumen de cambios y tendencia en el campo de la IG

\begin{tabular}{ll}
\hline & \multicolumn{1}{c}{ Cambios profundos } \\
\hline - & Leb 3.0 \\
- & La revolución del tiempo real [5] \\
- Nanotecnologías, miniaturización, sensorización y nuevos materiales [4] & \multicolumn{1}{c}{ Tractores } \\
- & IPV6, Telefonía 5G, expansión de las redes [4] \\
\hline & Blockchain [7] \\
\hline - Territorios/ciudades/casas/espacios inteligentes [1] [8] \\
- Internet de las Cosas, redes de sensores y redes de dispositivos y máquinas [1] \\
- Máquinas y dispositivos inteligentes, autónomos y colaborativos [8] \\
- Avanculos no tripulados [6] \\
- Industria 4.0 [8]
\end{tabular}


Continuación Tabla 1

\begin{tabular}{|c|c|}
\hline \multicolumn{2}{|c|}{ Tendencias en datos } \\
\hline $\begin{array}{ll} & \text { Web semántica [11] } \\
\text { - } & \text { BIM [8] } \\
\text { - } & \text { Big Data [1] [2] [5] } \\
\text { - } & \text { LiDAR [9] }\end{array}$ & $\begin{array}{ll}\text { - } & \text { Open Data, Linked Data }[11] \\
\text { - } & \text { Ciencia ciudadana y } \\
\text { "observation web" [6] } \\
\text { - } \quad \text { Coche conectado y mobile } \\
\text { mapping[6] }\end{array}$ \\
\hline \multicolumn{2}{|c|}{ Tendencias en $I G$} \\
\hline $\begin{array}{ll}\text { - } & \text { Captura masiva y barata [6] } \\
\text { - } & \text { Datos 4D nativos [9] } \\
\text { - } & \text { Desarrollo de geoservicios [9] } \\
\text { - } & \text { Administración digital [10] } \\
\text { - } & \text { Posición Indoor [1] }\end{array}$ & $\begin{array}{ll}\text { - } & \text { Localización como servicio [5] } \\
\text { - } & \text { SIG en tiempo real y en la nube } \\
& {[5]} \\
\text { - } & \text { Necesidad de análisis y accesos } \\
& \text { rápidos [5] } \\
\text { - } & \text { Aplicaciones trasnacionales y } \\
\text { globales [10]. }\end{array}$ \\
\hline
\end{tabular}

[1] Carpenter y Snell (2013); [2] Eldawy y Mokbel (2015); [3] Lee y Kang (2015);

[4] Richardson (2017), [5] Dangermond (2017), [6] NGAC (2016), [7] Meyer, (2018),

[8] Oesterreich y Teuteberg (2016), [9] Shukla (2014), [10] Kwang (2016)

La manera en que la IG llega al usuario posee en la actualidad una esencia dual, por un lado, la IG se representa mediante datos, que se transfieren para su explotación en local en el sistema del usuario. Pero por otro, la IG se utiliza en muchos casos a través de geoservicios pensados para explotar los datos en remoto mediante peticiones que se incorporan a la lógica de la aplicación. La tendencia de cara al futuro es que esta opción última sea la dominante. Por tanto, hablar de calidad de datos geográficos desde una perspectiva de usuario, deja de tener sentido pleno si no se liga a la calidad de los metadatos y servicios que permiten accederlos. Por otro lado, aunque las organizaciones se suelen considerar como un componente más de una IDE (GSDI, 2012), no se ha considerado hasta ahora suficientemente su calidad.

Se ha de considerar que la IG tiene un valor político significativo porque permite el establecimiento integrado de políticas en diferentes sectores (agricultura, transporte, desarrollo regional, medio ambiente). El reconocimiento de esta importancia al más alto nivel político se ha desarrollado de la mano de la preocupación creciente por el desarrollo sostenible, y ya fue incluido en el Tratado de Ámsterdam en 1997 como uno de los objetivos clave de la Unión Europea. Todos los objetivos de desarrollo sostenible de la ONU tienen una vinculación con lo geoespacial, ya sea de una manera más directa y evidente (p.ej. energía asequible, acción por el clima, vida de ecosistemas terrestres, ciudades y comunidades sostenibles, etc.), como de una forma más indirecta (p.ej. la justicia social y la igualdad de género por medio de la administración de tierras). En relación con la calidad de los datos espaciales, el informe de 2010, "Iniciativa de las Naciones Unidas sobre la Gestión Global de 
la Información Geoespacial” indica que, “...el incremento en las fuentes de datos, uno de los papeles clave de las agencias oficiales de cartografía para los siguientes cinco a diez años será, definir y mantener los estándares de calidad y los regímenes actuales de información con respecto de los datos que el gobierno requiriera para efectos de cumplir con sus operaciones". Por tanto, la buena planificación y ejecución de los objetivos de desarrollo sostenible requiere datos espaciales de calidad.

Este trabajo presenta un planteamiento en tres líneas, en primer lugar, se atenderá a la calidad de los datos en sentido estricto, en segundo término, a la calidad de los servicios web basados en ellos y, por último, a las organizaciones. Todas ellas están ligadas entre sí. La calidad de un servicio depende de la calidad de los datos asociados. La calidad de un productor de datos se evidencia en parte en la calidad de los datos que produce y los servicios que implementa. A su vez, se puede considerar que la calidad de estos dos últimos se ve afectada inevitablemente por la gestión de la calidad en el productor de datos. En cada parte, obviaremos los aspectos de la calidad ya considerados anteriormente.

Las bases conceptuales de la calidad de datos son básicamente las normas ISO 19100 que establecen cómo describir de manera normalizada la calidad de la IG, sus metadatos y especificaciones. En el caso de la calidad de servicios, consideraremos esencialmente los estándares OGC y las Normas de Ejecución para servicios de la Directiva INSPIRE, un intento muy serio de caracterizar la calidad de geoservicios, que se está llevando a cabo en un universo de más de 100.000 servicios. ${ }^{1} \mathrm{El}$ tratamiento de la calidad en los productores de datos se ofrece desde una perspectiva centrada en la IG y no en los sistemas de gestión de la calidad (p.ej. ISO 9001 o EFQM). ${ }^{2}$

De esta forma, el objetivo de este trabajo es revisar de una manera crítica el modelo actual relativo a la calidad de datos geográficos, geoservicios y organizaciones, para considerar todos los aspectos relacionados con la calidad. Junto a esta revisión crítica, la principal aportación que realiza este trabajo es indicar un conjunto de líneas de trabajo para la mejora e, igualmente esbozar nuevos campos de investigación relacionados con la calidad.

Se ha adoptado como perspectiva dominante la centrada en los productores de datos geográficos oficiales, dado el papel preponderante y protagonista que desempeñan en el campo de la Información Geográfica.

En consecuencia, con todo lo indicado, el documento incluye las distintas perspectivas (datos, servicios, organizaciones), dedicando a cada una de ellas un apartado. Finalmente se incluyen una discusión y unas conclusiones generales.

$1 \quad$ Véase $<\mathrm{http}: / /$ inspire-geoportal.ec.europa.eu/discovery/>.

2 Fundación Europea para la Gestión de la Calidad, <http://www.efqm.org/> 


\section{Calidad de datos}

La calidad de los datos es un aspecto que lleva recibiendo atención desde los inicios de la Cartografía como tecnología. Sin embargo, los primeros modelos o marcos conceptuales sobre todos los aspectos de la calidad que pueden concurrir en un conjunto de datos espaciales (CDE) es algo relativamente reciente. Fue en 1982 cuando en Estados Unidos se iniciaron los primeros trabajos bajo el auspicio del ACSM (American Congress of Surveying and Mapping). Así, se creó una propuesta de estándar (Moellering, 1987) donde se habla de adecuación al uso, de informes de la calidad y se establecieron cinco categorías de elementos de la calidad (linaje, exactitud posicional, exactitud de atributos, consistencia lógica y compleción). Es relevante indicar que todavía se sigue trabajando con estos elementos de la calidad, casi sin apenas cambios. Esta propuesta de estándar, algo modificada, fue adoptada como Federal Information Processing Standard 173 por el National Institute of Standards and Technology (NIST, 1994). De esa misma época es el manual de la Asociación Cartográfica Internacional (ICA) que, editado por Guptill y Morrison (1995), fue el catalizador de una mayor preocupación internacional sobre esta temática en ámbitos universitarios e investigadores.

Casi en paralelo, el European Committee for Standardization (CEN) estuvo trabajando en el desarrollo de una prenorma europea (pr EN 12652:1997) centrada en la calidad de los datos (CEN, 1997). Algo después, el comité técnico 211 de ISO (ISO/TC 211) estableció las normas internacionales ISO 19113:2002, ISO 19114:2003 y la especificación ISO/TS 19138:2006. Todas ellas constituyen la base de la norma internacional sobre calidad de datos geográficos ISO 19157:2013 (ISO 2013), actualmente en vigor. Los productos de datos deben tener una calidad adecuada a su propósito que esté modelada correctamente, para lo que se requieren unas especificaciones que orienten la producción y unos metadatos que informen adecuadamente de cara a su uso. Por tanto, desde la perspectiva de la calidad se deben abarcar tanto el modelo, como las especificaciones y los metadatos. A continuación se tratará sobre cada uno de ellos.

\section{Calidad: el modelo}

El modelo de calidad de datos actualmente más extendido a nivel internacional es el propuesto en la norma internacional ISO 19157. De manera resumida, se propone un modelo basado en distintos aspectos de la calidad, los denominados elementos de la calidad (p.ej. exactitud posicional absoluta, exactitud posicional relativa, comisiones y omisiones, corrección de la clasificación, etc.). Estos elementos son cuantitativos y para su evaluación la norma internacional ISO 19157 propone un conjunto de medidas normalizadas de calidad, y unos procesos básicos de evaluación. Con elementos de calidad y ámbitos concretos en los que se aplican, se pue- 
den crear las especificaciones de productos de datos (ISO 19131) e informar sobre la calidad de unos datos en sus metadatos (ISO 19115). Los elementos de calidad que se proponen son bastante limitados, pero ISO 19157 también propone un elemento denominado usabilidad, que permite soslayar, en parte, esa limitación. Además, esta norma también permite evaluar la metacalidad (la calidad de las evaluaciones de la calidad).

Este modelo presenta diferentes problemas. Desde una perspectiva centrada en las propias normas de ISO/TC211 no ha mantenido el criterio de retrocompatibilidad. Se ha cambiado un aspecto fundamental de la filosofía de ISO 19113, que era el poder definir nuevos elementos de la calidad, cosa que ya no es posible con ISO 19157. Por otra parte, este modelo está obsoleto. Desde 2003 hasta la fecha han aparecido numerosos avances en el campo de la calidad de datos, de las tecnologías de la información y de las necesidades de los usuarios que no se han tenido en cuenta, además, se han detectado numerosos fallos en ISO 19157. Empezando por lo último, es una característica común a las normas del ISO/TC211 de primera generación, las adoptadas entre el año 2000 y el 2007, el olvidar las imágenes y los datos malla. Esta circunstancia sigue presente en la norma ISO 19157, adoptada en 2013, que puede considerarse de segunda generación, pues no incluye elementos de calidad propios de imágenes. Igualmente, tampoco es totalmente adecuada para describir la calidad de metadatos, pues la calidad de campos como los de texto libre (p.ej. legibilidad, etc.) tampoco puede incorporarse adecuadamente a los elementos de calidad actualmente existentes (véase Ureña-Cámara et al., 2018). ISO 19157 está centrada en productos de datos, no tanto en datos, lo que dificulta su aplicación desde la perspectiva de los procesos actuales de generación de productos y publicación de servicios por integración de datos de muy distintas fuentes. Eso supone un gran inconveniente desde la perspectiva de la trazabilidad que se requiere en conjuntos de datos complejos en los que cada dato puede provenir de una fuente distinta (p.ej. un catastro en el que cada predio procede del levantamiento de un profesional diferente). Se requiere un grano más fino en el modelo, que cada elemento (p.ej. una instancia de punto, curva o relación) lleve una "mochila" propia con sus valores de calidad y que, por medio de agregación de los valores individualizados de instancias, se pueda pasar a dar un valor de calidad general a un CDE. Esta idea es la que define Ariza-López (2013a) como micrometadatos de calidad.

Algunos aspectos adicionales relativos a la calidad de un CDE no considerados en la norma ISO 19157 son: la posesión de algún tipo de certificación de la calidad, la consistencia con otros conjuntos de datos externos y un modelo de evolución temporal de la calidad. En relación con la certificación, que es la posibilidad de mantener algún sello de "estar certificado", este aspecto afecta tanto a las organizaciones, profesionales y productos. En ninguno de estos tres ámbitos existen esquemas de certificación. De cara al universo de aplicaciones no tripuladas que se 
avecina (p.ej. coches autónomos (Wikipedia, 2018a)), tendrán especial importancia tanto la integridad de los datos como con la trazabilidad, debido a la responsabilidad que se pueda derivar de ellos (EYGM, 2017). Integridad y trazabilidad de datos serán tratadas posteriormente. En relación con la consistencia con otros conjuntos de datos externos, Giordano y col. (1994) definían la confrontabilidad como la cualidad de poder integrar conjuntos de datos procedentes de fuentes distintas, algo estrechamente ligado con la interoperabilidad de datos (Ariza López, 2013b) y que se recoge ya en algunas Normas de Implementación de INSPIRE. Por otra parte, el mundo real es dinámico y los datos son registros que pueden quedar desfasados, por ello se requiere de un modelo de evolución temporal de la calidad, ligado al ciclo de vida de los objetos geográficos y de los datos que los representan. Así, la actualidad de dos conjuntos de datos de la misma fecha, uno de Hidrografía y otro de Carreteras, por ejemplo, puede ser muy diferente en función de la tasa anual de variación de objetos geográficos. En el caso mencionado, una vez transcurridos diez años, los datos de Hidrografía pueden resultar todavía válidos, mientras que los de carreteras pueden ser completamente inservibles. En Ariza-López (2013b) se puede encontrar una primera aproximación a un modelo temporal para la calidad de datos y cuya implementación directa sobre el modelo ISO es algo compleja si no se liga a la idea de micrometadatos de calidad.

Por otra parte, dentro del propio universo ISO han surgido otras normas que se refieren a calidad de datos como, por ejemplo, la de calidad de datos de productos (ISO/TS 8000-1:2011) que desarrolla un modelo distinto. Eso es una clara limitación de cara al futuro, por ejemplo, para la integración de datos de productos con datos espaciales por medio de modelos BIM (Building Information Model). En campos específicos, como el de la aviación, han surgido demandas de elementos de la calidad que no se han incorporado en el modelo ISO 19157, por ejemplo, la Organización de Aviación Civil Internacional establece en su Anexo 15 requisitos de integridad (grado de garantía en que no se han perdido o alterado ninguna referencia aeronáutica o sus valores). Desde la perspectiva de los usuarios, también existe un problema en la descripción y evaluación de la calidad: el modelo actual está centrado en productos de datos de carácter general y es poco entendible para ellos. En la actualidad son muy diversos los tipos de datos que se crean (como p.ej. datos LiDAR, fotos, vídeos, multimedia, escenarios, vistas estereoscópicas, 3D, 4D, datos enlazados...), los usuarios que los usan y las aplicaciones en las que se utilizan. Junto a esta diversidad existe un problema añadido: el gran salto entre lo que se especifica y evalúa en calidad, que presenta la perspectiva de los productores tradicionales y se modela con ISO 19157, y lo que realmente necesitarían los usuarios (adecuación al uso).

La calidad de los datos es una preocupación generalizada, pero una vez que la calidad relativa a los datos se va asentando surge la necesidad de conocer y ofrecer 
información más detallada y transparente sobre la calidad de los datos relativos a la calidad; este es el concepto de metacalidad: la calidad de los datos de calidad. Esta necesidad, apuntada de manera pionera por la prenorma europea de calidad ENV 12656 (CEN, 1998), se ha incorporado en la norma ISO 19157 por medio de tres elementos de metacalidad: confianza, representatividad y homogeneidad.

La evaluación de la metacalidad conlleva mayores exigencias que la de la calidad, y por ello hacen falta avances conceptuales, metodológicos y herramientas específicas, pero también la aparición de perfiles profesionales más especializados en la calidad de los datos espaciales, y también sistemas de certificación sobre la aplicación de las normas tanto a nivel de productos, procesos, organizaciones y capacidades de los profesionales. La metacalidad es algo que existe fuera del ámbito geomático desde hace mucho tiempo. Uno de los ámbitos donde la idea de metacalidad está presente es, a modo de ejemplo, el campo metrológico, donde existe una jerarquía de laboratorios que van asegurando encadenadamente la calidad de los instrumentos de medición. Incluso en la Norma Internacional ISO 9001, las exigencias sobre los equipos de medición, inspección y ensayo suponen asegurar la metacalidad instrumental y de las medidas realizadas con ellos.

Por otra parte, la falta de información relativa a la metacalidad es un riesgo en sí misma. La caracterización de unos parámetros de calidad de un CDE sin información sobre su metacalidad puede ocasionar situaciones nefastas. Por un lado, si el producto es realmente bueno, pero existe una subestimación de la calidad del CDE, al no disponer de información sobre la calidad de las estimaciones de la calidad, lo lógico será no utilizar ese recurso, con las consecuencias de despreciar una IG de valor en una toma decisiones. Además, si realmente se pretende producir un CDE con la calidad descrita, se puede estar produciendo un derroche de recursos innecesario. Por otro lado, si el producto es realmente malo y existe una sobreestimación de la calidad del CDE, lo lógico será utilizar ese recurso, con las consecuencias de incorporarlo en una toma decisiones. Pero aquí el productor no es consciente de que necesita aplicar mayores recursos o rigor para obtener la calidad deseada. Una producción muy descentralizada obliga a un mayor esfuerzo en la caracterización de los productores debido a su mayor número y diversidad y, como consecuencia, un esfuerzo muchísimo mayor en todo lo relativo a la metacalidad. Esto lleva, de manera directa, a la necesidad de automatizar las evaluaciones de la calidad y a tener alguna valoración sobre la confianza de los productores. Ambos aspectos son necesidades claras de cara a futuro y un campo de investigación inmediato.

\section{Calidad de metadatos y trazabilidad}

Hasta hace pocos años la cartografía era producida en forma de monopolio u oligopolio (instituciones cartográficas nacionales), y era utilizada mayoritariamente por técnicos, científicos y militares, los cuales tenían una formación que les capacitaba 
para su uso, y además disponían de información relevante sobre los productos. Sin embargo, junto a la revolución TIC, el advenimiento de la globalización y la Web 2.0, se ha producido una clara democratización en el uso y producción de datos geoespaciales (Kumar, 2000). Eso ha generado que nuevos grupos sociales accedan al uso de estos productos sin la formación e información adecuadas, en un entorno de mayor abundancia y volatilidad de la oferta de productos de datos. En esta situación, los metadatos $\mathrm{y}$, en particular, la descripción de la calidad son un elemento clave.

En un paradigma de producción descentralizada de IG, para que el sistema sea realmente eficaz en el uso de los recursos existentes, se requiere que toda la IG generada disponga de metadatos y que éstos estén orientados a los usuarios (Comber et al., 2010). Los metadatos han de permitir conocer la existencia de un CDE y evaluar, a priori, la posibilidad y conveniencia de usarlo (p.ej. extensión, calidad, licencias, etc.). Podemos afirmar que lo que no está catalogado no existe, pues no se podrá encontrar en la red ni explotar correctamente. Por otra parte, consideramos que lo que no está bien catalogado constituye una fuente de riesgo, pues puede inducir usos inadecuados.

Si bien los metadatos son simples datos, se incluyen en este análisis pues tienen un carácter singular, son un elemento clave y, en general, presentan numerosos problemas específicos desde la perspectiva de su calidad. Por otra parte, en un modelo orientado a objeto, datos y metadatos deben estar encapsulados y gestionarse como un todo, en la medida de lo posible, a lo largo de todo el ciclo de vida de los datos, desde su adquisición hasta su explotación final. La norma multiparte ISO 19115 establece el modelo de metadatos actualmente más extendido en el campo de la IG. Se trata de un conjunto de normas de gran extensión y complejidad, con gran cantidad de elementos opcionales y condicionales. Con ese conjunto de partes se han normalizado, en gran medida, la estructura y formato físico de los metadatos. Sin embargo, la gran cantidad de opciones en la norma, la proliferación de perfiles $\mathrm{y}$, sobre todo, el que su aplicación no haya estado acompañada de especificaciones rigurosas y criterios sobre cómo generar sus contenidos, hacen que los contenidos generados al aplicarla no estén suficientemente normalizados en la práctica.

Desde la perspectiva de informar sobre la calidad de manera más pormenorizada, la norma ISO 19157 propone un informe independiente de calidad. Sin embargo, el informe tiene formato libre y no está normalizada ni siquiera una estructura mínima para su redacción. Generar estructuras de informes para aplicaciones concretas y ayudas para su aplicación es una tarea clara a bordar en un futuro inmediato. Además de esa falta de normalización, posiblemente los cuatro problemas principales de la calidad de metadatos sean la omisión, la falta de actualidad, la falta de rigor y la falta de criterio. La omisión es la falta de metadatos, a este respecto todavía son muchos los conjuntos de datos, y en general los recursos, que existen 
sin que se disponga de unos metadatos adecuados. Esa carencia también afecta a contenidos que, si bien son opcionales en el modelo ISO 19115, realmente tienen una gran importancia y utilidad, pero no se suelen incluir en aras de disponer de unos metadatos mínimos.

La falta de actualidad es consecuencia del modelo que se ha implantado para la gestión de los metadatos. Según Lopez-Pellicer (2018), poseen un modelo cuasi analógico, en el sentido de que, si bien están almacenados en digital, la concepción con la que se manejan es casi la de una ficha bibliográfica en papel, pues no se usa la potencialidad de los enlaces. Además, el modelo ISO 19115 es muy pesado, ello dificulta su aplicación a la hora de crear metadatos automatizados para conjuntos de datos creados por integración de datos de múltiples fuentes. Se requiere un modelo de metadatos a nivel de datos que sea capaz de poder agregarse de manera automatizada, y de permitir la trazabilidad.

Podemos definir la trazabilidad como la capacidad de conocer el historial, ubicación y trayectoria de un producto o lote de productos a lo largo de la cadena de suministros en un momento dado, a través de unas herramientas determinadas (Wikipedia, 2018b). La trazabilidad de los productos es algo que está asumido en muchos campos de la industria (p. ej. automovilística, espacial, alimentaria, electrónica, etc.) y que también han de llegar al campo de la IG. La trazabilidad de datos ya se ha introducido en otros campos como el master data management (MIKE2, 2018). Por ello cabe esperar que en el caso de la IG sea una demanda que se consolide y tome fuerza en un futuro próximo. En este caso, la generación de $\mathrm{CDE}$ por integración de otros $\mathrm{CDE}$ supone un reto tecnológico importante en la gestión de la trazabilidad. La falta de trazabilidad genera numerosos riesgos en los productos. El no conocer los suministradores, procesos, operadores, métodos, etc., deja al productor sin capacidad de exigir responsabilidades, y sin capacidad de mejorar el producto de cara al futuro. En la industria (p.ej. de componentes electrónicos) la falta de trazabilidad está estrechamente ligada al denominado mercado informal o paralelo (grey market), donde es usual que los productos y procesos no cumplan realmente las especificaciones del producto de marca. Por ello, las grandes multinacionales no dejan de controlar la trazabilidad de sus productos y suministros, por lo que, por analogía, una producción de IG de calidad internacional debe cuidar ese aspecto.

Por otra parte, tanto el modelo de metadatos actual, como las herramientas para su generación y gestión, transmiten una perspectiva tradicional, en la que se siguen procedimientos similares a los analógicos pero en un entorno digital. Así, no se toma ventaja de los enlaces/vínculos, de los datos enlazados, de los sistemas de sindicación, etc., que facilitan las tecnologías actuales y, por tanto, el planteamiento es poco adecuado para el mantenimiento actualizado de los metadatos a lo largo del tiempo. Esta situación genera que cualquier evolución, tanto en los datos como en 
todo lo relacionado institucionalmente con ellos (p.ej. en los responsables, en las direcciones de contacto, etc.), sea muy pocas veces recogido con suficiente agilidad como para mantener los metadatos suficientemente actualizados.

Por falta de rigor nos referimos a los numerosos problemas de contenido de muy diversa índole: falta de exactitud posicional en las coordenadas de los BoundingBox, inconsistencias dentro de sus propios contenidos, valores fuera de rango, equivocaciones en las clasificaciones asignadas, resúmenes y propósitos de escasa legibilidad o de valor casi nulo, faltas de ortografía, topónimos confundidos, linajes que aportan muy poco, etc. (véase Ureña-Cámara y col. 2018, que presenta una evaluación de la calidad de los metadatos de la IDE de España).

Finalmente, a menudo se detecta la falta de un criterio uniforme y homogéneo que haga que los metadatos completados por diferentes personas, o por una misma persona en distintos momentos, sean comparables y, por lo tanto, útiles para hacer búsquedas y comparar resultados. Esa situación se debe esencialmente a la falta de profesionales documentalistas especializados en la gestión de información geográfica que asuman esas tareas. Es lo que hace que se eche de menos la figura del curador de datos. ${ }^{3}$

Efectivamente, junto al modelo y las herramientas, posiblemente otro factor que ha influido en la baja calidad de los metadatos es que no hayan sido generados por profesionales en esa materia, muchas veces se han dejado para el final del proceso productivo y en manos de operadores no expertos. Además, si bien el modelo ISO 19157 se puede aplicar a la calidad de los metadatos, lo cierto es que no es algo usual por lo que las especificaciones de los metadatos muchas veces han estado huérfanas de parámetros de calidad que definieran los niveles deseados en el producto de metadatos.

Como consecuencia de las deficiencias y problemas descritos se vislumbran algunas tendencias que pueden contribuir a elevar la calidad de los metadatos en un futuro:

- Simplificación de los modelos de metadatos, reteniendo los ítems más útiles y relevantes. Por ejemplo, los metadatos INSPIRE están formados por 19 ítems para datos y 17 para servicios, frente a los más de 200 de la norma ISO 19115$1: 2014$.

- Aparición progresiva de la figura del curador de datos geoespaciales, encargado de la catalogación, preservación y curación de conjuntos de datos.

- Automatización del proceso de generación de metadatos, probablemente aplicando inteligencia artificial y aprendizaje automático (machine learning) e información de flujos de trabajo automatizados.

- Navegabilidad de metadatos de recursos acoplados, es decir que sea posible relacionar mediante los oportunos ítems de enlace, los metadatos de unos 
datos con los metadatos del servicio de visualización que los publica, éstos a su vez con los metadatos del servicio de descarga a través del cual se pueden conseguir y así sucesivamente.

- Gestión integral y encapsulada de datos y metadatos en una aproximación orientada a objeto que gestione los metadatos necesarios en cada fase del ciclo de vida de los datos, desde la adquisición hasta el almacenamiento como datos históricos.

\section{La calidad y las especificaciones}

En el campo de la industria está claro que no se puede obtener un buen producto sin unas buenas especificaciones. Es más, desde un punto de vista conceptual se puede decir que un producto no está bien definido si los usuarios no disponen de sus especificaciones. En este sentido, se dispone de la norma ISO 19131 relativa a las especificaciones de producto de datos. Consideramos que esta norma debiera ser aplicada por todas las organizaciones públicas que poseen competencia en la producción de datos geoespaciales, si bien su grado de implementación es bastante escaso. En su aplicación ocurre muchas veces como con los metadatos, que se adopta una postura de mínimos que hace que los productos no estén completamente especificados. A menudo el problema no es la propia norma, sino la falta de documentos técnicos adicionales que la complementen pues, en general, hay poca propensión a detallar y especificar bien los procesos de producción por el coste de gestión que ello supone. Puesto que se utiliza el modelo de calidad descrito anteriormente, en la aplicación de ISO 19131 también se dan las limitaciones mencionadas respecto a la adecuación al uso. Centrados en este punto, y dado que numerosos conjuntos de datos son utilizados por los usuarios en procesos concretos que pertenecen a dominios de aplicación específicos, y muy diferentes entre sí, en las especificaciones se hace cada vez más necesario el incluir determinaciones de la calidad funcional requerida. La especificación de calidad funcional supone dar el salto desde la calidad de los datos (p.ej. exactitud posicional horizontal absoluta de $5 \mathrm{~m}$ al 95\%), a algo más aplicado y satisfactorio (funcional) en una aplicación concreta (p.ej. que el 95\% de las longitudes de los cauces derivados del modelo de elevaciones son adecuadas para su uso hidrológico directo en un tipo de aplicaciones concretas).

En un marco donde las TIC aseguran la interoperabilidad de muchos tipos de datos, los datos espaciales siguen ofreciendo numerosos problemas a ese respecto. El marco desarrollado por el TC211 de ISO no deja de ser un marco orientado a la interoperabilidad, pero es muy general y se ha centrado casi en exclusiva en las interoperabilidades técnica y sintáctica. Así, se requieren más esfuerzos en busca de una mayor interoperabilidad semántica que permita el uso integrado y sinérgico de los conjuntos de datos provenientes de distintos productores. La mejor manera 
de conseguir la interoperabilidad, ya sea dentro de un mismo país o en una región (p.ej. Europa o el continente americano), es partir de diseños por medio de unas buenas especificaciones. En esta línea, de cara al futuro, se requiere de mucho mayor esfuerzo en la definición de especificaciones de productos concretos para dominios de aplicación concretos, en lo que los procesos federados o confederados serán de gran relevancia.

Las especificaciones adolecen prácticamente de los mismos problemas y deficiencias que los metadatos. En esta línea, buena parte de las ideas esbozadas y conclusiones relativas a metadatos (p.ej. figura del curador, machine learning, etc.) son aplicables a las especificaciones.

En una primera y somera aproximación, consideramos que los aspectos más relevantes sobre los que hay que centrar la atención para que sean realmente útiles a los usuarios son: i) normalización, ii) compleción y corrección, iii) aplicabilidad y iv) inclusión de la adecuación al uso del producto (calidad funcional). En esta última línea, se requieren medidas de la calidad que no sean "datocéntricas" (como las propuestas en ISO 19157) sino "usocéntricas", y una usabilidad definida de una manera más rica. Por tanto, se pueden considerar, al menos, dos líneas de investigación e innovación todavía no iniciadas hasta el momento: a) la profundización en medidas de calidad de datos centradas en usos concretos (casos de uso), y b) establecimiento de modelos que permitan describir y cuantificar de manera normalizada la calidad de unas especificaciones de producto, una vez que se ha descrito adecuadamente el propósito con el que se producen los datos.

\section{Calidad de datos y su aplicación}

Para concluir este apartado dedicado a la calidad de los datos, los metadatos y las especificaciones, se presenta un pequeño estudio realizado el 1 de abril de 2018 consultando la información disponible en 19 páginas web de otras tantas organizaciones nacionales responsables de la cartografía oficial (National Mapping Agencies) del continente americano. Así, se ha encontrado que tan sólo en 11 ocasiones $(\approx 58 \%$ ) se publican metadatos de los datos y servicios disponibles, que sólo una organización $(5,3 \%)$, publica información de calidad de sus datos más allá del linaje y que solo en 6 ocasiones (32\%), se publica información descriptiva de los productos de datos geográficos disponibles, si bien en 4 ocasiones se denomina "Documentación técnica" y únicamente en un caso, se presenta como especificaciones.

La pregunta que surge de modo natural es ¿Por qué, si bien la generación y publicación de metadatos está relativamente extendida, no ocurre lo mismo con las especificaciones y sobre todo con la calidad de la IG? Consideramos que hay varias razones que pueden ser responsables de la escasa implantación de procesos de determinación de la calidad: 
- Carencias en la producción de datos geográficos y en su puesta al día, lo que hace que en ocasiones los responsables prefieran invertir todos sus recursos en la producción de información y no detraer parte de ellos para hacer determinaciones de la calidad.

- Realizar determinaciones de la calidad suele ser costoso, consume recursos, requiere un modelo de calidad y expertos que no siempre existen y, además, devuelve en ocasiones resultados no deseados o al menos, digamos políticamente poco correctos, por lo que la motivación para realizar los correspondientes trabajos es baja.

- No existen, al menos en conocimiento de los autores, organizaciones que se dediquen a certificar la conformidad con la norma ISO 19157, lo que supone un desincentivo para su aplicación, al no ser un mérito reconocido por terceros.

- En la producción de otros tipos de información gubernamental (p.ej. estadística o meteorológica), no se realizan determinaciones y publicación de parámetros de calidad, por lo que los gobiernos no están habituados a aplicar modelos de calidad a la información que producen.

Sin embargo, el considerar un modelo de calidad de datos geográficos, e implementarlo, tiene ventajas estratégicas para los productores de datos oficiales:

- Puede marcar una diferencia positiva entre las fuentes de datos oficiales y la información de otros proveedores de datos y servicios no oficiales (p.ej. OpenStreetMap, GeoNames o Google Maps).

- El tener una calidad bien descrita y controlada parece uno de los requisitos exigibles a los datos fundamentales de un país, generados con el propósito de servir para georreferenciar todo tipo de datos temáticos.

- Matiza muy conveniente la responsabilidad legal de los productores de datos geográficos oficiales, e informa convenientemente a los usuarios de sus limitaciones. Sin información de calidad, los usuarios ingenuos pueden tener la impresión, y de hecho la tienen a menudo, de que los datos son perfectos y no tienen errores, con lo que pueden formular quejas e incluso emprender acciones legales si sufren daños o perjuicios debido a errores en los datos geográficos. Sin embargo, si se publican datos geográficos acompañados de una descripción de su calidad, por un lado, se difunde el mensaje de que los datos no son perfectos y tienen sus limitaciones. Por otro lado, el productor es responsable nada más (y nada menos) que de garantizar la calidad especificada.

Como se deduce del panorama mostrado, la situación no puede considerarse como avanzada o positiva. Son muchos los inconvenientes que existen y, posiblemente, pocas las recompensas. Consideramos que la introducción de esquemas de certificación específicos en el campo de la calidad de datos espaciales, para las organizaciones, productos y profesionales, podría ser un aliciente para la mejora en este campo. Siempre habría organizaciones interesadas en mostrar su excelencia y 
ejercerían de ejemplo para las demás. Otra posible línea, es la implantación de métodos de comparación (benchmarking) que puedan ser aplicados por terceros para evidenciar las distintas situaciones de cada productor (p.ej. organización, país, etc.). El modelo de madurez país relativo a la calidad de la IG propuesto por Xabier y col. (2017), dentro del proyecto de asistencia técnica del IPGH "Diagnóstico de la situación actual sobre las metodologías y procedimientos empleados para la evaluación de la calidad de la Información Geográfica” ofrece una opción de gran interés.

\section{Calidad de servicio}

En ciencias de la computación, un servicio es un componente abierto, autodescrito y que admite la composición rápida y de bajo coste de aplicaciones distribuidas. El proveedor de servicios es la organización que implementa, brinda soporte técnico y comercial, y proporciona las descripciones de un servicio. Estas descripciones pueden incluir información sobre el servicio, su interfaz, ubicación, comportamiento e incluso calidad. Los servicios web proporcionan una manera sistemática y ampliable para la interacción entre aplicaciones, que está construida sobre protocolos web existentes y basadas en estándares abiertos.

La calidad de un servicio (QoS, de Quality of Service) es compleja debido a tres características exclusivas de los servicios: intangibilidad, heterogeneidad e inseparabilidad de la producción y el consumo, sin embargo, es algo muy necesario, tanto en los servicios que se nos ofrecen como personas físicas (p.ej. un servicio de restauración, o alojamiento), como en los servicios web que utilizamos junto con datos espaciales. Qué supone la calidad de servicio en el ámbito web es algo sobre lo que no existe un acuerdo generalizado. Sin embargo, como muestra la Tabla $2,{ }^{4}$ son muchos los aspectos $\mathrm{y}$, por tanto, términos, que se utilizan para tratar sobre este asunto.

Tabla 2

Términos relacionados con la calidad de servicio

\begin{tabular}{|c|c|c|c|c|c|c|c|c|c|c|c|c|c|c|}
\hline $\begin{array}{l}\text { Términos usados para } \\
\text { la calidad }\end{array}$ & 1 & 2 & 3 & 4 & 5 & 6 & 7 & 8 & 9 & 1011 & 12 & 13 & 1415 & Total \\
\hline Availability & & $\mathrm{x}$ & $\mathrm{x}$ & $\mathrm{x}$ & $\mathrm{x}$ & $\mathrm{x}$ & $\mathrm{x}$ & $\mathrm{x}$ & $\mathrm{x}$ & & $\mathrm{x}$ & $\mathrm{x}$ & & 10 \\
\hline Reliability & $\mathrm{x}$ & & $\mathrm{x}$ & $\mathrm{x}$ & $\mathrm{x}$ & $\mathrm{x}$ & $\mathrm{x}$ & $\mathrm{x}$ & $\mathrm{x}$ & $\mathrm{x}$ & $\mathrm{x}$ & & & 10 \\
\hline Security & & $\mathrm{x}$ & $\mathrm{x}$ & $\mathrm{x}$ & $\mathrm{x}$ & $\mathrm{x}$ & $\mathrm{x}$ & & $\mathrm{x}$ & & $\mathrm{x}$ & & $\mathrm{x}$ & 9 \\
\hline $\begin{array}{l}\text { Capacity or } \\
\text { throughput }\end{array}$ & & $\mathrm{x}$ & $\mathrm{x}$ & & $\mathrm{x}$ & $\mathrm{x}$ & & $\mathrm{x}$ & $\mathrm{x}$ & & $\mathrm{x}$ & $\mathrm{x}$ & & 8 \\
\hline Response time & & $\mathrm{x}$ & $\mathrm{x}$ & & $\mathrm{x}$ & $\mathrm{x}$ & $\mathrm{x}$ & $\mathrm{x}$ & $\mathrm{x}$ & & & & $\mathrm{x}$ & 8 \\
\hline
\end{tabular}

4 Para evitar posibles confusiones con los términos, se dejan en inglés. 
Continuación Tabla 2

Términos usados para la calidad

$$
\begin{array}{lllllllllllllllll}
1 & 2 & 3 & 4 & 5 & 6 & 7 & 8 & 9 & 10 & 11 & 12 & 13 & 14 & 15 & \text { Total }
\end{array}
$$

Performance

Accuracy

Scalability or

Adaptability

Transaction Integrity

Accessibility

Assurance or

consistency

Completeness

Compliance

Cost

Interoperability

Latency

Popularity

Empathy

Reputation

Responsiveness

Successability

Usability

$\begin{array}{lll}\mathrm{x} & \mathrm{x} & \mathrm{x} \\ & & \mathrm{x}\end{array}$

$\mathrm{x}$

X $\quad \mathrm{X} \quad \mathrm{X}$

$\mathrm{X} \quad \mathrm{X}$

$\mathrm{x} \quad \mathrm{X}$

$\mathrm{X}$

$\mathrm{X}$

$\mathrm{X}$

$\mathrm{x}$

$\mathrm{X}$
$\mathrm{X}$

$\mathrm{X}$

$\mathrm{x} \quad \mathrm{x}$

$\mathrm{X}$

$\mathrm{x}$

$\mathrm{X}$

$\mathrm{x}$

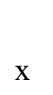

$\mathrm{x}$

$\mathrm{x}$

$\mathrm{x}$

$\mathrm{x}$

$\mathrm{x}$

$$
\mathrm{X}
$$

$\mathrm{X}$

$\begin{array}{ll}\mathrm{X} & 7\end{array}$

7

4

4

4

3

3

$\mathrm{x} \quad 3$

$\begin{array}{ll}\mathrm{x} & 3\end{array}$

3

3

3

3

2

2

2

2

2

[1] Zeithaml y col. (1990); [2] Menascé ( 2002); [3] Brahnmath y col. (2002);

[4] Mani y Nagarajan (2002); [5] Lee y col. (2003); [6] DeLone y McLean ( 2003); [7] Kang (2007); [8] Al-Masri y Mahmoud (2008); [9] Kim y col. ( 2011);

[10] Yang y col. ( 2006); [11] Subbiah y col. ( 2007); [12] INSPIRE (2008);

[13] Gao y col. (2009); [14] Khaled y col. (2010); [15] Wu y col. (2011).

Fuente: Xabier y col. 2013.

Indudablemente, debe incluir la conformidad con el estándar seleccionado y, en una primera aproximación, lo que se suele entender como calidad de servicio operacional. En el marco INSPIRE la QoS se define mediante tres aspectos: 
- Disponibilidad, que se define como la probabilidad de que el servicio de red responda a una petición correcta. En INSPIRE se mide como un porcentaje de disponibilidad anual. Para determinarlo se deben realizar peticiones cada 6 minutos, como especifica el marco INSPIRE (Comisión Europea, 2009), que deben estar perfectamente definidas y descritas. En el Reglamento INSPIRE (Comisión Europea, 2009) se exige al menos un 99\% de disponibilidad anual excepto tiempos de parada técnica anunciados con una semana de antelación. Hay que hacer notar que para un mismo tanto por ciento de disponibilidad, la exigencia es mucho mayor cuanto menor es el tiempo de medida (Tabla 3).

- Rendimiento, que se define como velocidad de respuesta. Se mide en el marco INSPIRE mediante el tiempo de respuesta. Es el tiempo que se tarda en recibir el primer bit de respuesta a una consulta dada. Para calcular medias anuales de tiempos de respuesta de cada servicio se suelen emplear las mismas peticiones que sirven para determinar la disponibilidad. El marco INSPIRE (Comisión Europea, 2009) establece un límite de 5 segundos para un servicio WMS de visualización y 3 segundos para un servicio CSW de catálogo.

- Capacidad, que se define como el número máximo de peticiones simultáneas de servicio que se responden con un rendimiento garantizado. Por tanto, es la posibilidad de responder un pico de peticiones, como por ejemplo 20 peticiones por segundo durante un minuto, manteniendo el tiempo de respuesta establecido (Comisión Europea, 2009).

Tabla 3

Tiempo máximo de parada semanal, mensual y anual para una misma disponibilidad

\begin{tabular}{llll}
\hline & \multicolumn{1}{c}{ Semanal } & \multicolumn{1}{c}{ Mensual } & \multicolumn{1}{c}{ Anual } \\
\hline $90,00 \%$ & $17 \mathrm{~h} 5 \mathrm{~min}$ & $73 \mathrm{~h}$ & 36 días $12 \mathrm{~h}$ \\
$95,00 \%$ & $8 \mathrm{~h} 31 \mathrm{~min}$ & $36 \mathrm{~h} 30 \mathrm{~min}$ & 18 días $6 \mathrm{~h}$ \\
$99,00 \%$ & $1 \mathrm{~h} 45 \mathrm{~min}$ & $7 \mathrm{~h} 20 \mathrm{~min}$ & 3 días $15 \mathrm{~h}$ \\
$99,50 \%$ & $51 \mathrm{~min}$ & $3 \mathrm{~h} 39 \mathrm{~min}$ & 1 día $19 \mathrm{~h} 40 \mathrm{~min}$ \\
$99,99 \%$ & $1 \mathrm{~min} 3 \mathrm{~s}$ & $4 \mathrm{~min} 30 \mathrm{~s}$ & $53 \mathrm{~min}$ \\
\hline
\end{tabular}

Hay que decir que en ocasiones faltan criterios claros y objetivos para elegir el periodo de medida tanto del rendimiento como de la disponibilidad. En ambos casos, la práctica más frecuente es determinar su valor anual, y adquirir algún tipo de compromiso de calidad con los usuarios en cuanto a mantener un umbral mínimo en cada caso. Elegir un periodo anual tiene la ventaja de que posibles fallos o episodios de baja calidad, si no son muy acusados, se compensan a lo largo del resto del año. El inconveniente es que, ante un episodio de muy baja calidad, hay que esperar todo un año para conseguir que el indicador arroje un buen resultado. 
En ese sentido, para informar mejor al usuario, es siempre recomendable aplicar el principio de transparencia y publicar datos de rendimiento y disponibilidad diarios o semanales para complementar la información que dan los indicadores anuales.

Por otro lado, entendemos que, si la disponibilidad y el rendimiento se definen adecuadamente, la capacidad es redundante. Efectivamente, si un servicio en producción durante un año completo satisface el nivel de disponibilidad exigido y, al mismo tiempo, es capaz de proporcionar un tiempo de respuesta medio adecuado durante el $10 \%$ del tiempo diario que soporta mayor carga de trabajo, demuestra tener capacidad suficiente para soportar las condiciones de explotación reales.

Por último, otras fuentes (Mani y Nagarajan, 2002; Branmath et al., 2002 y Lee et al., 2003) consideran otros aspectos adicionales, de los que se indican los más importantes en la Tabla 4.

Tabla 4

Aspectos de la QoS adicionales a los considerados en el marco INSPIRE

\begin{tabular}{lccc}
\hline Aspecto de la QoS & W3C (1) & IBM (2) & Brahnmath (3) \\
\hline Escalabilidad & $\mathrm{X}$ & & $\mathrm{X}$ \\
Robustez & $\mathrm{X}$ & & \\
Control de excepciones & $\mathrm{X}$ & & $\mathrm{X}$ \\
Exactitud & $\mathrm{X}$ & $\mathrm{X}$ & \\
Integridad & $\mathrm{X}$ & $\mathrm{X}$ & \\
Accesibilidad & $\mathrm{X}$ & & \\
\hline
\end{tabular}

[1] Lee y col. (2003), [2] Mani y Nagarajan (2002), [3] Brahmmath y col. (2002).

En donde (Tabla 4):

- La escalabilidad es la capacidad de un servicio de incrementar los recursos informáticos disponibles, de manera que su capacidad aumente cuando aumenta el número de peticiones por unidad de tiempo.

- La robustez representa la capacidad de un servicio de funcionar correctamente cuando le llegan peticiones erróneas o con parámetros disparatados y poco habituales.

- El control de excepciones alude a la calidad de un servicio de describir de manera adecuada en un mensaje de error el motivo por el que no puede dar respuesta a una petición incorrecta.

- La exactitud se define como la tasa de respuestas erróneas devueltas por el servicio web ante peticiones correctas.

- La integridad es la capacidad del servicio de evitar accesos no autorizados a los datos y software almacenados, lo que tiene que ver con la seguridad, y la capa- 
cidad de mantener la consistencia de los datos durante las actualizaciones, lo que es importante en los servicios transaccionales.

- La accesibilidad representa si el servicio web es capaz o no de responder a las peticiones de los usuarios.

Puede entenderse fácilmente que no todos los aspectos de calidad de servicio mencionados son independientes entre sí y, por ello, puede ser de interés centrarse en los que presentan mayor independencia. Bajo esta idea, consideramos que pueden ser más relevantes: la robustez, el control de excepciones y la exactitud. No obstante, es necesario investigar en los aspectos de la calidad que más relevancia tengan para los servicios geográficos: estudiar qué medidas y métodos de determinación son viables y qué aspectos es razonable y útil considerar para mejorar su explotación.

Lo indicado hasta ahora en este apartado son aspectos tradicionales de calidad de servicios, pero el uso diario de los geoservicios destaca la existencia de otros muchos aspectos de su calidad que debieran ser tenidos en consideración y que, hasta la fecha, no han sido suficientemente tratados en la literatura. Así, a continuación, se presentarán algunos aspectos relevantes que ofrecen una perspectiva novedosa que debe ser abordada por la calidad.

Un aspecto no considerado hasta ahora es la calidad del contenido de la respuesta debida no a los datos, suficientemente cubierto por los aspectos considerados de calidad de datos, sino a cómo presenta los datos el servicio. Es decir, cómo está definido y configurado el servicio web. Por ejemplo, para un Servicio Web de Mapas esto incluye entre otros aspectos: a) el sistema de referencia de coordenadas elegido, b) la distribución de datos en capas, c) las escalas a las que es visible la información de cada capa, d) la calidad del documento de capacidades (capabilities), e) la calidad de la representación, etc. Algunos de estos aspectos pueden desplegarse muchísimo, así la calidad de representación abarcaría la leyenda, los colores elegidos, los símbolos puntuales, lineales y superficiales, los tipos de letra y su tamaño, etc. Muchos de estos aspectos son cruciales en geoservicios y, sin embargo, poco tenidos en cuenta habitualmente en los estudios.

Lo lógico sería disgregar la calidad de un servicio en calidad de cada una de las operaciones que admite y hablar así de calidad de GetMap, calidad de GetCapabilities y calidad de la operación opcional GetFeatureInfo, si está implementada.

Especialmente importante nos parece la elección de una paleta de colores adecuada, basada no solo en el tono sino también en el brillo, adaptada para todo tipo de daltonismo, más adaptada a la visualización en pantalla que al papel y que tenga especialmente en cuenta las pantallas de los teléfonos inteligentes.

En otros servicios, como el servicio de catálogo, este aspecto incluiría también la calidad de cada una de sus operaciones, entre las que habría que tener especialmente en cuenta la cosecha de metadatos (harvesting), que resulta crítica porque 
permite sincronizar catálogos y, en la práctica, origina muchas dificultades cuando los catálogos están implementados sobre programas diferentes.

También los servicios de descarga de ficheros exigen parámetros de calidad específicos, como la velocidad de descarga, la tasa de interrupciones, la variedad de formatos disponibles y la calidad de la descripción del servicio.

Por último, para mencionar otro ejemplo de servicio básico y muy usado en una IDE, los servicios de nomenclátor (gazetteer) pueden presentar un amplio abanico de características de las que depende su calidad, como: opciones de autocompletado y corrección de errores en los términos de búsqueda, búsquedas inteligentes que tengan en cuenta la posición del usuario y su historial de peticiones, posibilidad de admitir cualificadores borrosos (p.ej. cerca de, lejos de, en la dirección de...), búsquedas de términos similares y relacionados, ordenación de resultados, etc.

De nuevo estamos ante aspectos poco estudiados que constituyen candidatos a tener en cuenta a la hora de definir los programas de investigación e innovación a acometer en el campo de la IG, más concretamente en el campo de las IDE.

\section{Calidad de productores de datos}

Los apartados anteriores, centrados en los datos y servicios, tienen una perspectiva técnica centrada en la IG y en las TIC. En este apartado, incluimos uno de los factores más importantes: las organizaciones donde se produce la IG en un contexto de descentralización, como es frecuente en la actual producción de datos espaciales. Ninguno de los aspectos tratados en los demás apartados de este documento es independiente de la organización y del contexto en los que se materializan, por lo que también es relevante su análisis.

Antes de avanzar, en línea con las ideas de Cinnamon (2014), conviene aclarar que consideramos que la producción de datos espaciales, presente y futura, no se puede entender como un sistema binario con polos en IGV y agencias cartográficas tradicionales. Por el contrario, debe entenderse como un continuo que puede alentar el desarrollo de hibridaciones que aprovechen lo mejor de cada uno de los participantes. Desde esta perspectiva, los aspectos de la calidad son aún más críticos que antes y, por ello, el enfoque de adoptamos no es el que tradicionalmente se considera, centrado sobre las organizaciones responsables de cartografía oficial, donde los sistemas de gestión de la calidad (p.ej. aplicación de ISO 9001, Barrot y Pla, 2018), los modelos de calidad (p.ej. IECA 2013a, 2013b) y las herramientas (Mesterton y Kivekäs, 2018), son los aspectos sobre los que suele centrarse la atención. En este trabajo nos preocupa la IG y por ello el análisis se centrará en los que, a nuestro modo de ver, consideramos son los mayores riesgos inmediatos en este paradigma de producción descentralizada de IG en el que cualquier agente social se convierte fácilmente en productor de datos geoespaciales: 
- Producción: no comprometerse con todo lo que significa.

- Acceso: no facilitar accesos de calidad.

- Trazabilidad y metacalidad: no mantener el linaje e información sobre la calidad de la calidad.

- Preservación: no asegurar el uso futuro.

A continuación se presentan en mayor detalle cada uno de estos aspectos.

\section{Producción: no comprometerse con lo que significa}

Podemos pensar que actualmente la producción de IG es sencilla pues está muy facilitada por las numerosas y cada vez más baratas tecnologías de captura. Pero esto no es del todo cierto. La producción de datos geoespaciales en la actualidad requiere más compromisos que los que se asumían anteriormente. Los modelos de datos que se están proponiendo y utilizando son cada vez más sofisticados, por ejemplo, hoy en día es usual hablar de: ciclo de vida de los fenómenos del mundo real, ciclos de vida de los datos, ciclos de vida de los productos, dato único, identificadores únicos permanentes, sellos temporales, datos enlazados, sindicación de datos espaciales, microdatos, reglas de implementación, etc.

La captura es mucho más sencilla que antes, pero ahora el valor añadido es también mucho mayor que antes. Se trata de una producción mucho más tecnificada, compleja y dependiente del conocimiento, que ha de ser escalable y también capaz de evolucionar más ágilmente que nunca para estar al nivel de exigencia de las tecnologías TIC que utilizan los usuarios. Realmente no se producen datos, se producen modelos digitales que ofrecen distintas perspectivas virtuales de la realidad.

La producción IGV presenta notables problemas en lo relativo a la calidad de los datos (Senaratne y col., 2017), pero este no es el único problema de la producción descentralizada. La producción ha de suponer, ahora más que nunca, un compromiso mantenido a lo largo del tiempo y una visión sistémica de todo el proceso de producción. Desde esa perspectiva de la producción descentralizada de los datos, los principales riesgos que vislumbramos se relacionan con el fraccionamiento y debilidad de los productores con poca experiencia, en ocasiones aquellos que se han incorporado en los últimos años a la producción de datos espaciales alentados por las actuales facilidades para la captura de datos y para ofrecer geoservicios. Entre otros, los problemas principales pueden ser:

- Relativos a la producción continuada

- Falta de perspectiva temporal. Básicamente ocurre en agentes noveles, que consideran la creación de datos desde una perspectiva episódica y aislada. La creación de datos espaciales debe suponer un compromiso a largo plazo, pero si bien es lógico que ese compromiso no sea asumido por productores 
neófitos, ocasionales o voluntarios, también es verdad que en organizaciones oficiales no siempre se asume con el debido rigor.

- Falta de viabilidad institucional. No siempre existe un mandato legal explícito para la producción de datos, lo que no impide que se aborde. Así, hoy en día se producen datos geoespaciales por los más variados motivos, algunos coyunturales, como una bajada de costes y se considera una tendencia a seguir. Pero, en ocasiones, la organización no asume ese compromiso de cara al futuro por lo que, al menor cambio, se deja de producir.

- Relativos a los aspectos tecnológicos

- Exigencias tecnológicas crecientes. La generación/captura puede ser cada vez más sencilla y económica pero el mantenimiento, preservación, inclusión de ciclos de vida, creación de unos buenos metadatos, evolución tecnológica sostenible de los datos, etc., suponen cada vez más exigencias añadidas a la producción. Sólo unos pocos con financiación y personal altamente cualificado pueden acometer con éxito su incorporación al modelo de producción.

- Acceso mediante servicios. En el paradigma de producción de la cartografía tradicional, e incluso de datos digitales sobre soportes físicos (p.ej. DVD, CD-ROM, etc.), el producto se materializaba en un recurso físico accesible, aunque el productor hubiera dejado de existir. En el paradigma actual y futuro, los datos se ofrecen y utilizan por medio de servicios web, si es el productor quien los ofrece y ese productor deja de existir la cuestión es ¿se seguirán ofreciendo esos servicios?

- Relativos a la fragmentación de la producción.

- Idiosincrasia. Si bien las organizaciones tradicionales productoras de datos espaciales son bien distintas y presentan un comportamiento distinto debido a sus experiencias vitales, personal, know-how, etc., con la llegada de nuevas formas de producción, como es la IGV, aparecen patrones muy distintos que hay que saber entender para utilizar bien sus productos. Así, si bien la IGV se ha puesto de moda como forma de crear datos espaciales (p.ej. OpenStreetMap, GeoNames), es de destacar que los datos creados por la mayoría de esos movimientos tienen notables limitaciones (p.ej. falta de cubrimiento homogéneo de todo el espacio geográfico, grandes niveles de omisión, no existe una clasificación única de los elementos, calidad semántica baja, etc.).

- Interoperabilidad. La fragmentación de la producción y aparición de nuevos productores supone graves riesgos para la interoperabilidad de los datos si no se refuerzan las acciones de coordinación. En un marco de producción 
descentralizada se requiere un mayor compromiso de todas las partes para que los datos sean interoperables y así conseguir la sinergia que posibilita el uso conjunto. El aspecto de la interoperabilidad abarca numerosas perspectivas y ya ha sido tratado.

\section{Acceso: no facilitar accesos de calidad}

En la actualidad los productos de datos espaciales, se ofrecen en forma de servicios vía web, por lo que también se ha de pensar en éstos como elementos críticos. En el contexto TIC es obligado considerar un paradigma descentralizado en la oferta de servicios de acceso a datos espaciales. En este sentido, dado que en otro apartado se tratará sobre la interoperabilidad, y que en el epígrafe anterior se ha esbozado el mantenimiento de los servicios a lo largo del tiempo, este epígrafe se centra en la gestión de la calidad de servicio. Muchos productores de IG, muchos de ellos con escasos recursos (p.ej. pequeñas municipalidades, organizaciones no gubernamentales, particulares, etc.), podrán llegar a generar IG de calidad e interés para la comunidad; pero la capacidad de ofrecer servicios con accesos de calidad (p.ej. disponibilidad, tiempo de respuesta, seguridad, fiabilidad, etc.), puede quedar fuera de su alcance. De esta manera, el riesgo para la calidad de los servicios de datos geoespaciales consiste en no tener una disponibilidad efectiva del recurso por limitaciones en la prestación del servicio debido a que no todos los proveedores de datos geoespaciales tienen capacidad económica ni tecnológica para ofrecer servicios de calidad.

\section{Interoperabilidad: no posibilitar la utilización y uso legal}

Bajo nuestro punto de vista, los mayores riesgos de la producción descentralizada son los relativos a la interoperabilidad. La interoperabilidad es la base de las TIC y por ello es la piedra angular de las IDE y de la Geomática 3.0 tal como se ha definido anteriormente. Según Rodríguez y col. (2013), la interoperabilidad es la idea clave hacia la que se orientan todas las actividades de normalización y estandarización en el campo de la IG. ISO la define formalmente (ISO, 2008) como: "capacidad para comunicar información, ejecutar programas o transferir datos entre unidades funcionales de manera que el usuario solo necesite tener un conocimiento escaso o nulo sobre las características particulares de tales unidades" [ISO/IEC 2382-1:1993].

En el campo de las IDE una definición de interés es la que proporciona la Directiva INSPIRE (Parlamento y Consejo Europeos, 2007): "posibilidad de combinación de los conjuntos de datos espaciales y de la interacción de servicios, sin intervención manual repetitiva, de forma que el resultado sea coherente y se aumente el valor añadido de los conjuntos y servicios de datos". 
Para conseguir la interoperabilidad se requieren acuerdos entre las partes. Estos acuerdos se materializan en forma de normas internacionales, especificaciones, estándares, reglamentos, etc. Por lo que la interoperabilidad está ligada íntimamente a las acciones de coordinación y liderazgo que resulten en documentos como los indicados anteriormente.

La interoperabilidad abarca distintos campos que van desde lo más tecnológico o duro, hard (p.ej. formatos, sistemas de referencia, semánticas, etc.) a lo más suave, soft (p.ej. aspectos institucionales, aspectos legales de licencias de uso, etc.) y no por ello menos costoso. La falta de interoperabilidad en los datos es posiblemente el riesgo más evidente y que más hemos sufrido en el trabajo con IG a lo largo de las últimas décadas. Ejemplo de esto son los problemas de intercambio de formatos, los problemas de integración de modelos de datos y semánticas distintas, los problemas de interoperabilidad debidos a discrepancias posicionales, etc. Siguiendo con lo estrictamente relativo a los datos, las técnicas de conflación o también denominadas de integración o fusión de datos (Xavier et al., 2016), son las encargadas de solventar estos problemas (p.ej. conflación vertical, conflación horizontal, (RuizLendínez y col. 2011)). Pero también hay otros aspectos no menos relevantes, como las limitaciones legales, que pueden surgir del uso CDE con modelos de licencia que no resulten compatibles. En el caso de la producción descentralizada llevada cabo por las administraciones, también se puede hablar de interoperabilidad institucional, la cual puede abarcar muchas facetas que impidan el uso conjunto efectivo de datos.

En principio, la descentralización se puede considerar un movimiento de incremento de la entropía y, como tal, que va en contra de alcanzar acuerdos técnicos, que son la base de la interoperabilidad. Un mayor número de productores de datos espaciales, muchos de ellos de carácter sectorial, otros amateurs, otros esporádicos, etc., tendrán tendencias naturales a crear "desorden". Por ello, la producción descentralizada de datos geoespaciales supone notables riesgos de interoperabilidad si no existe una labor eficaz de liderazgo para alcanzar consensos que la favorezcan. Sin embargo, en contraposición a todo lo indicado previamente, ejemplos como INSPIRE nos demuestran que un liderazgo fuerte permite aprovechar la variedad y diversidad de los sistemas descentralizados para aprovechar lo mejor de cada uno creando un proyecto conjunto. Una de las claves del éxito en el liderazgo de un sistema descentralizado es facilitar especificaciones y herramientas que resulten cómodas para que nadie se salga de la corriente principal.

\section{Preservación: asegurar el uso futuro de los datos espaciales}

La debilidad del almacenamiento digital ha llevado a acuñar el término digital dark age como aquella posible situación futura en la que sería imposible o muy difícil 
acceder a documentos del pasado por estar almacenados en soportes y formatos digitales obsoletos. A nivel mundial existe verdadera preocupación por este asunto y se están tomando iniciativas vinculadas con el desarrollo de las denominadas einfraestructuras. La IG es un tipo de contenido digital de gran valor económico y que interesa conservar por motivos legales, administrativos, científicos, culturales, sociales y de toda índole (Ariza-López et al., 2012).

La calidad está relacionada con el uso adecuado, satisfactorio y fructífero. Sin embargo, un factor limitativo del uso y beneficio futuro del uso de la IG es su preservación o curación. Para ello se requiere un conjunto de metadatos especiales (distintos de los propuestos en ISO 19115 o en Dublin Core) que son los que deben permitir la caracterización de los contenidos digitales y, a partir de ellos, permitir la gestión de los procesos de preservación. En esta línea, el TC/211 de ISO está desarrollando la norma internacional ISO 19165 sobre preservación de datos y metadatos. Junto a los metadatos para la preservación también se requiere una organización y gestión adecuada del sistema que, en parte, puede ser parecido al paradigma IDE. Este marco debe cubrir adecuadamente:

- La necesidad de incluir la perspectiva de preservación y explotación futura de los datos desde el diseño de los productos de datos espaciales mediante el diseño de un ciclo de vida de producto.

- Considerar la necesidad de establecer distintos alcances temporales en la preservación, como por ejemplo el corto (5 años), medio (20 años) y largo plazo (100 años), según la importancia de los datos.

- Establecer un conjunto de metadatos especiales pensados para la preservación e integrables con las normas ya existentes.

- Establecer las bases para la prueba de los sistemas de preservación que puedan implantarse.

- Establecer las bases para una infraestructura federada de preservación a nivel nacional.

- Evaluar la calidad (eficacia y eficiencia) de los sistemas de preservación.

La producción descentralizada ocasiona productores y producciones más dinámicas y efímeras, y heterogeneidad en la preservación. Estos dos aspectos dificultan la preservación futura de la IG y suponen un claro riesgo de ocultación o desaparición de conjuntos de datos, lo que origina, a su vez, claros retos para la investigación.

\section{No afrontar una verdadera transformación digital}

Para acabar este apartado, consideramos importante retomar la idea de la necesidad verdadera transformación digital de las organizaciones dedicadas a la producción de datos espaciales y en la manera de servirlos. El contexto que definen, por la parte de 
generación de datos, la existencia de múltiples sensores, la internet de las cosas, los espacios inteligentes, los BIM, la realidad virtual y la realidad aumentada, etc., y por la parte de las aplicaciones los vehículos no tripulados para el transporte de personas y mercancías, la agricultura de precisión, la robótica y mecatrónica, etc., hace pensar que realmente se requiere otra forma de producir datos espaciales y que para ello es necesario un cambio radical. Esta transformación digital debe ir orientada a asegurar conjuntos de datos únicos (single source of Truth, SSOT), dotados de identificadores únicos persistentes, globales y enlazados, que den soporte continuado (espacio y tiempo) a las múltiples aplicaciones inteligentes y robotizadas (automatizadas).

Desde esta perspectiva, se podría pensar que serán exitosas aquellas organizaciones que sean capaces de implementar sistemas de servicios que ofrezcan sus datos SSOT para que sean utilizados masivamente por terceros (individuos, empresas, organizaciones, administraciones, etc.) en la sociedad más allá de las TIC.

\section{Discusión}

De manera paralela a la necesidad de un desarrollo sostenible, la sociedad actual se encuentra sometida a un continuo de revoluciones tecnológicas (nanotecnologías, miniaturización, tiempo real, sensorización, etc.), y de cambios tractores (Ciudades inteligentes, Internet de las cosas, datos enlazados, vehículos autónomos, Industria 4.0 , etc.), y cambios sociales, que nos llevan a un paradigma donde los datos son el combustible del futuro. En este paradigma los datos espaciales son un elemento clave, imprescindible y de valor en alza. Estos cambios afectan a la forma de generar datos geoespaciales, a su uso y a las exigencias sobre ellos, estableciendo nuevos retos para la calidad de esos datos geoespaciales, de los geoservicios y de las organizaciones que los producen.

En los apartados anteriores se ha realizado una descripción y crítica de la situación actual, al igual que algunas propuestas sobre qué conviene investigar y trabajar de cara al futuro. Como se ha indicado, son visiones parciales, pero lo que realmente consideramos más crítico es la falta de una visión sistémica, global, y sostenible en el tiempo, de todos los aspectos intervinientes (datos, servicios y organizaciones), en la que los datos geográficos fundamentales (datos geoespaciales cuyo propósito es servir para georreferenciar otros datos, que serán temáticos) y no solo la calidad, sino también la eficacia en solucionar los problemas de calidad que los usuarios reclamen, están llamados a ser cada vez más esenciales. En una sociedad sostenible, como la que se propugna desde la ONU, hacen falta datos geoespaciales de calidad, adaptables, actualizados y coherentes entre sí, como parte de un modelo sostenible de creación, sistematización, uso y preservación de datos espaciales. 
A nuestro entender, esta visión sistémica y sostenible no existe, ni con una perspectiva global ni más parcial o sectorial. Indudablemente, lo que se sugiere es complejo y será difícil de plasmar, pero no se parte de cero: como se ha evidenciado en el texto, existen numerosos elementos (p.ej. calidad de datos, calidad de servicios, calidad de los productores de datos, modelo de aseguramiento de la calidad de datos espaciales, etc.). Todos estos elementos, convenientemente extendidos y adaptados, pueden ser parte de la solución para conformar el modelo que se requiere. Así, por ejemplo, consideramos que el modelo propuesto en IECA (2013a) para el aseguramiento de la calidad en la producción de datos geoespaciales, puede ser adaptado y extendido para incluir aspectos de calidad de metadatos, geoservicios y de datos preservados. Indudablemente, realizar la propuesta queda fuera de las posibilidades de este trabajo, pero lo que sí podemos y debemos hacer es preguntarnos ¿por qué no existe aún ese modelo? La respuesta no es sencilla ni única, pero apuntando posibles problemas, también apuntaremos posibles soluciones. Bajo nuestro punto de vista son varios los factores:

- Complejidad. Se trata de un tema realmente complejo. Requiere de un trabajo interdisciplinar y de expertos. Existen numerosos perfiles de expertos, pero justamente el de expertos en calidad de datos geoespaciales, ya sea en su vertiente de evaluación de la calidad o de gestión de la calidad, no es nada abundante. Además, no se conoce ningún foro que los convoque o haya convocado para hacerlos trabajar de manera conjunta en esta temática.

- Escasos datos abiertos. Existe una escasa implantación de políticas de datos abiertos. Según establece el Global Open Data Index, tan solo el 11\% de los conjuntos de datos que considera claves se publican como datos abiertos, ${ }^{5}$ porcentaje que baja hasta el $10 \%$ en los datos geoespaciales más esenciales. ${ }^{6}$ Una apertura general de datos podría ser un revulsivo para que los usuarios utilizasen y comparasen masivamente los datos geoespaciales, y se promoviera su calidad.

- Idiosincrasia de la calidad. Para los productores de cartografía oficial, que son los que más necesitan de la visión que se propone aquí, la calidad es un aspecto sobre el que existe poca transparencia. Preguntarles sobre la calidad de sus datos, sobre los procesos de aseguramiento, control, etc., no siempre es bien recibido. La calidad es algo interno, oneroso y sobre lo que prefieren pasar de puntillas. No se invierte en formación específica y tampoco se dedican esfuerzos internos $\mathrm{y}$, mucho menos, existe diálogo y coordinación externa para avanzar. Por su parte, para las empresas privadas que viven de los datos y servicios geoespaciales (p.ej. TomTom, Where, Google y otras), muchos aspectos de la calidad forman parte de su know-how y, como es lógico, no están dispuestos a

$5<$ https://index.okfn.org/>.

$6<$ https://index.okfn.org/dataset/map/>. 
compartirlos. Por otra parte, en las agencias cartográficas oficiales hay una escasa implantación de una cultura de calidad de producto final. Pocas categorías de datos producidos por los gobiernos van acompañadas de información de calidad; como ejemplo significativo podemos mencionar los datos estadísticos que, a pesar de que no suelen ir acompañados de información de calidad son utilizados para todo tipo de decisiones de gran trascendencia.

- Prioridades de normalización. Uno de los problemas fundamentales reside en cómo se realiza la normalización y, en concreto, las normas de datos geoespaciales, y las barreras para su utilización. Los comités técnicos de normalización son muy especializados y están focalizados sobre visiones muy parciales, cuando la calidad es algo más transversal. Así, aún bajo el paraguas de un mismo cuerpo de normalización, la cooperación entre comités no siempre es tarea fácil. Centrados en el caso del TC211 de ISO, y después de 24 años de existencia, podemos afirmar que su actividad sobre la calidad de datos no ha sido todo lo amplia que podría haberse esperado, y ello no significa crear normas internacionales para todo, existen otros documentos ISO que también pueden tener gran valor. Así, la calidad de las imágenes no se ha abordado, la calidad de los geoservicios tampoco, el Anexo D de medidas de calidad apenas se ha extendido, la exactitud posicional no se ha abordado, etc. Junto a lo ya indicado, NGAC (2017) reconoce la existencia de notables barreras a la utilización de estándares, entre ellas la falta de herramientas conformes, la falta de iniciativas para hacerlos funcionar, la no inclusión en los programas académicos, etc.

- Comunidades de productores. Los temas de calidad son complejos y no siempre una única organización puede abordar proyectos complejos, por ello es importante trabajar con otras organizaciones que compartan los mismos problemas. Sin embargo, salvo excepciones (p.ej. Eurogeographics), no existe un alto grado de asociacionismo de instituciones productoras de cartografía oficial, en las que se aborden temas técnicos como el aquí planteado, y donde se propongan soluciones convenidas entre todas ellas. Lo mismo ocurre a nivel de profesionales especializados en calidad de datos. No tenemos conocimiento de ninguna asociación que agrupe los intereses de profesionales expertos en la materia y que realice propuestas técnicas.

- Falta de responsabilidad legal. Hasta la fecha de hoy, la producción de datos oficiales, salvo para los casos náutico y aeronáutico, no tiene una exigencia de responsabilidad que lleve a los productores a esforzarse en todo lo relacionado con la calidad, la trazabilidad, preservación, etc. de sus datos. Un marco legislativamente más exigente sería un revulsivo para dedicar más esfuerzos a la temática con una visión más global y sistémica. Posiblemente, este marco pueda llegar de mano de la regulación de sectores como los vehículos autónomos. 
- Falta de certificación. La falta de aliciente de un reconocimiento externo (p.ej. sello, acreditación, certificación, etc.) que destaque frente a la sociedad la bondad del trabajo realizado también puede actuar como factor negativo a la hora de tomar iniciativas. Así, como se ha indicado, un modelo de madurez, desarrollado a nivel de país u organización, y reconocido por tercera parte, podría ser muy positivo. Por un lado, ofrecería una guía de lo que hay que hacer (lo que se evalúa) y por otra, la oportunidad de que los esfuerzos fueran reconocidos.

\section{Conclusiones}

En este trabajo se ha presentado un análisis centrado en la calidad de los datos, la forma en que se ofrecen (los servicios) y quienes los ofrecen (las organizaciones). Cada una de estas perspectivas es complementaria y no debe abordarse sin una visión conjunta. Sin embargo, a pesar de la extensión del trabajo, son numerosos los aspectos que no han sido tratados.

La IG, su producción y puesta en "servicio", se ven afectadas por los numerosos cambios y tendencias tecnológicos y sociales que tienen lugar, muchos de los cuales suponen verdaderas revoluciones respecto a las formas de hacer y de entender la IG de hace unos años, y suponen numerosos retos desde las perspectivas de la calidad que interesan en este trabajo.

Centrados en la calidad de datos, podemos concluir que existe un modelo de calidad consolidado conceptualmente (ISO 19157), pero escasamente aplicado, como se ha demostrado por el estudio realizado en las páginas web de diversas agencias cartográficas nacionales. Este modelo está muy limitado en sus posibilidades respecto a los retos inmediatos que se plantean tanto en tendencias de datos como de IG (Tabla 1). El grano, la inclusión del tiempo, la trazabilidad, elementos y medidas de la calidad usocéntricos, etc. son aspectos que no están bien resueltos en la actualidad.

La calidad de los datos se evalúa poco y se han apuntado posibles causas de ello. Evaluar la calidad de los datos es tanto más necesario cuanto más tecnificado y automatizado sea el entorno de los datos espaciales (p.ej. vehículos no tripulados, agricultura de precisión, etc.). Considerando los conjuntos de datos oficiales y fundamentales de cada país, se debe tender a una calidad aplicada, que realmente sea entendible por los que utilizan esos datos. Por otra parte, se puede minimizar el coste de las evaluaciones por medio de varias líneas de actuación, entre ellas, i) seleccionando el conjunto mínimo pero esencial de aspectos, medidas y métodos de la calidad que ofrezcan buen balance coste-beneficio; ii) desarrollando métodos automáticos o semiautomáticos de determinación de la calidad (p.ej. Xavier y col. 2015a y 2015b). 
La calidad de los metadatos es algo poco tratado y, por tanto, desconocido, a pesar de su gran importancia para el funcionamiento de los servicios. Su creación podrá ser automatizada en breve por medio de la Inteligencia Artificial aplicada a procesos y flujos de trabajo, documentos, etc. Existen campos de metadatos cuya calidad puede ser automatizada y evaluada al $100 \%$, pero otros campos relevantes como los resúmenes o el propósito apenas han sido evaluados (Ureña-Cámara y col. 2018). El modelo ISO 19115 es muy pesado y farragoso. Los metadatos están desligados de los datos y de su calidad, y eso es una grave limitación de cara al uso de datos simples, no de conjuntos de datos, para lo cual se requieren los que hemos denominado micrometadatos. Aspectos como la trazabilidad y navegabilidad requieren de perspectivas de implementación distintas a las actuales para ser funcionales. La creación y mantenimiento de metadatos con un verdadero carácter de enlazado es otro reto actual todavía no resuelto.

Las especificaciones de los productos de datos son un elemento clave de la calidad, pero su uso está muy limitado, muchas veces no están acompañadas de documentación técnica que les dé sentido. Además, se usan especificaciones de la calidad datocéntricas y no usocéntricas, lo que hace que los productos no queden bien especificados. Se utilizan descripciones de la calidad propias de la IG anterior a los SIG y no de descripciones de la calidad basadas en casos de uso concretos en dominios de aplicación determinados. No hay un método ni modelo para evaluar la calidad de las especificaciones de productos de datos, lo cual también impide mejorar (lo que no se mide, no se mejora).

En cuanto a la calidad de servicios web, consideramos que se han producido avances técnicos y realizaciones prácticas de monitorización continua de la calidad de servicio suficientemente aceptables. Como aspectos tradicionales de la calidad a evaluar, creemos que sería suficiente con tener en cuenta, como ya hemos dicho, la disponibilidad y el rendimiento, y quizás se podría añadir la estabilidad, que tiene que tendría en cuenta el número de caídas de un servicio, no solo el tiempo que dura cada caída. En este campo serían necesarios llevar a cabo estudios detallados que corroborasen o refutasen con datos objetivos esas conclusiones. Sin embargo, como se ha indicado, hay numerosos aspectos (p.ej. calidad de las operaciones, presentación de los resultados de las peticiones, etc.) que siendo importantes todavía no han sido difundidos ni estandarizados.

En lo relativo a las organizaciones en un marco de producción descentralizado y muy democratizado, se han identificado diversos riesgos que ponen en entredicho la calidad sostenida en cuanto a la producción de datos y su acceso a largo plazo cuando se trata de productores sin un mandato legal claro o de tipo esporádico. Centrados en las agencias cartográficas oficiales, un reto crítico es no afrontar la verdadera transformación digital de la sociedad que nos está llevando a un mundo digital que se configura como una realidad virtual, en muchos casos más allá de una 
simple copia del mundo realidad. En la mayoría de los casos, todavía no se han desarrollado modelos de datos y de producción federada basados en SSOT, que permitan un espacio inteligente (territorio inteligente + ciudad inteligente + edificios inteligentes + etc.) de carácter continuo, donde los datos enlazados, los datos de sensores, los datos de la internet de las cosas, los datos estadísticos, los datos administrativos (p.ej. licencias urbanísticas, licencias de cultivos, etc.), utilicen la IG fundamental por medio de identificadores únicos globales persistentes como soporte de una web realmente semántica y basada en datos enlazados.

Como se ha indicado en el apartado de discusión, según nuestra experiencia sería necesario un modelo que aporte una visión más sistémica, global y sostenible en el tiempo para gestionar todos los aspectos aquí señalados, pero ese modelo no existe. En la sección de discusión se han indicado algunos aspectos que requieren nuestra atención para mejorar la situación.

Finalmente, queremos indicar que para avanzar en la línea de superar las limitaciones actuales y de afrontar los retos que se han indicado, se hace especialmente crítico y necesario contar con personal técnico bien formado en todos estos campos. Por ello la formación, en sus distintos niveles (p.ej. grados, postgrados, especializaciones, cursos cortos, etc.) es un elemento clave que nos debe preocupar y debe ser cuidado. En esta línea, ayudaría en gran medida el establecer un conjunto de pénsum o planes de estudio que orientaran a los agentes formativos sobre los contenidos a desarrollar. Igualmente, el establecimiento de esquemas de certificación de personas ayudaría a dar mayor nivel a los técnicos con responsabilidades en este campo (p.ej. evaluación de la calidad). Junto a lo anterior, y dado que la base de la mejora es la comparación, consideramos necesario disponer de modelos de madurez que permitan a cada organización compararse con un estándar (que evolucionará a lo largo del tiempo), tal que en todo momento sepa a qué nivel de madurez corresponde su manejo de la calidad de datos, de los metadatos, de adopción de datos abiertos etc. Consideramos que, desde el IPGH se deberían liderar estas dos actividades tan necesarias en el marco panamericano.

Hemos de concluir indicando que trabajar y preocuparse por la calidad de los datos geográficos, de los geoservicios y productores de datos es trabajar y preocuparse para que se dispongan de mejores bases para la selección de alternativas, el diseño, ejecución, seguimiento y mejora de políticas, planes y proyectos que consoliden el alcance de los objetivos de desarrollo sostenible en cada uno de los países del mundo.

\section{Bibliografía}

AENOR (2003). UNE 66175 Sistemas de gestión de la calidad - guía para la implantación de sistemas de indicadores, Madrid, España, Asociación Española de Normalización. 
Al-Masri, E. and Mahmoud, Q. (2008). "Toward Quality-Driven Web Service Discovery", en IT Professional, 10, 24-28.

Ariza-López, F.J. (2013a). Calidad de la Información Geográfica: perspectivas de futuro, en CIMA INEGI, México.

Ariza-López, F.J. (ed.) (2013b). Fundamentos de evaluación de la calidad de la información geográfica, Jaén, España, Servicio de Publicaciones de la Universidad de Jaén.

Ariza-López, F.J.; Ariza-López, R.M.; Ureña Cámara, M.A.; Cortés José, J. y Ureña López, L.A. (2012). "Preservación de la Información Geográfica: Perspectivas y situación en España”, en GeoFocus 12, 171-200.

Barrot, D. and Pla, M. (2018). "ISO 9001 for spatial data: ICGC experience", en International Workshop on Spatial Data Quality, Valletta (Malta), Eurogeographics, $\quad<$ https://eurogeographics.org/wp-content/uploads/2018/06/3SDQ2018-12.pdf $>$.

Brahnmath G.J.; Raje, R.R.; Olson A.; Auguston M.; Bryant B.R. and Burt C.C. (2002). "A quality of service catalog or software components", en Proceedings of the Southeastern Software Engineering Conference, Huntsville, AL, USA, 513-20<http://www.dtic.mil/dtic/tr/fulltext/u2/a492809.pdf $>$, consultado el 01 de abril de 2018.

Carpenter, J. and Snell, J. (2013). Future Trends in geospatial information management: the five to ten-year vision, United Nations Committee of Experts on Global Geospatial Information Management.

CEN (1998). ENV 12656:1998 Geographic information - Data description - Quality. Brussels, Belgium, Comité Européen de Normalisation.

Cinnamon, J. (2014). "Deconstructing the binaries of spatial data production: Towards hybridity", en The canadian Geographer 59(1). DOI:10.111/cag.12119.

Comber, A.J.; Fisher, P.F. and Wadsworth, R.A. (2010). "User-focused metadata for spatial data, geographical information and data quality assessments", en $10^{\text {th }}$ AGILE Conference, Aalborg, Denmark, Association of Geographic Information Laboratories in Europe.

Comisión Europea (2009). Reglamento (CE) 976/2009 de la Comisión, de 19 de octubre de 2009 por el que se ejecuta la Directiva 2007/2/CE en lo que se refiere a los servicios de red, http://eur-lex.europa.eu/legalcontent/ES/TXT/PDF/?uri=CELEX:32009R0976\&from=ES, consultado el 24 de junio de 2018.

Dangermond, J. (2017). Five GIS Trends Changing the World according to Jack Dangermond, President of Esri. <http://geoawesomeness.com/five-gis-trendschanging-world-according-jack-dangermond-president-esri/>, consultado el 24 de junio de 2018. 
Dans, E. (2010). Todo va a cambiar. Barcelona, España, Editorial Deusto. Disponible en $<$ https://www.todovaacambiar.com/>, consultado el 24 de junio de 2018.

DeLone, W.H. and McLean E.R. (2003). "The DeLone and McLean model of information systems success: A ten-year update", en Journal of Management Information Systems 19, 9-30.

Eldawy, A. and Mokbel, M.F. (2015). "The era of big spatial data", en 31st IEEE International Conference on Data Engineering Workshops, Seoul, South Korea. DOI: 10.1109/ICDEW.2015.7129542.

EYGM (2017). The evolution in self-driving vehicles Trends and implications for the insurance industry, EYGM Limited.

Gao S.; Mioc D. and Yi X. (2009). "The measurement of Geospatial Web Service quality in SDIs", en Proceedings of the 17th International Conference on Geoinformatics. Fairfax, VA, USA, IEEE.

Giordano, A. and Veregin, H. (1994). Il controllo di qualitá nei sistemi informative territoriali. come valuare e mantenere l' accuratezza del database. Venezia, Italy, Il Cardo.

González-Campos, M.E.; Bernabé-Poveda, M. y León Pazmiño, M.F. (2017). “Metodología para evaluar la usabilidad del visualizador de mapas del geoportal IDE de Ecuador", en GeoFocus 19, 109-127.

Goodchild, M.F. (2007). "Citizens as sensors: the world of volunteered geography", en GeoJournal 69(4), 211-221. DOI:10.1007/s10708-007-9111-y.

IECA (2013). NTCA 01-002 Modelo Aseguramiento Calidad productos IG, Instituto de Estadística y Cartografía de Andalucía, Sevilla, España.

IECA (2013). NTCA 01-003 Modelo de Calidad para la IG en Andalucía. Instituto de Estadística y Cartografía de Andalucía, Sevilla, España.

INSPIRE (2008). Network Services Architecture, Version 3.0. Infrastructure for Spatial Information in Europe, $<$ http://inspire.jrc.ec.europa.eu/reports/ImplementingRules/network/D3_5_INSP IRE_NS_Architecture_v3-0.pdf $>$.

ISO (2008). ISO/TS 19104:2008 Geographic Information - Terminology. Geneva, Switzerland, International Organization for Standardization.

ISO (2013). ISO 19157:2013. Geographic information. Data quality. Geneva, Switzerland, International Organization for Standardization.

ISO/IEC (2005). TR 9764:2005 Information technology - Guidelines, methodology and reference criteria for cultural and linguistic adaptability in information technology products. Geneva, Switzerland, International Organization for Standardization.

Kang, Y. (2007). "Extended Model Design for Quality Factor Based Web Service Management", en Proceedings of the Future Generation Communication and Networking. Jeju, South Korea, IEEE, 484-487. 
Karpik, A.P. and Musikhin, I.A. (2016). "Research and practical trends in geospatial sciences", en The International Archives of the Photogrammetry, Remote Sensing and Spatial Information Sciences, Volume XLI-B6, 2016, XXIII ISPRS Congress, 12-19 July 2016, Prague, Czech Republic.

Khaled R.; Tayeb L. and Servigne, S. (2010). "Geospatial Web Services Semantic Discovery Approach Using Quality", en Journal of Convergence Information Technology 5, 28-35.

Kim, E.; Lee, Y.; Kim, Y.; Park, H.; Kim, J.; Moon, B.; Yun, J. and Kang, G. (2011). Web Services Quality Factors, Version 1.0. Burlington, MA, USA. Organization for the Advancement of Structured Information Standards (OASIS). $<$ http://docs.oasis-open.org/wsqm/WS-Quality-Factors/v1.0/cs01/WSQuality-Factors-v1.0-cs01.html>, consultado el 24 de junio de 2018.

Kumar, N (2000). "Automation and Democratization of Cartography: An Example of a Mapping System at CEM, University of Durham", en The Cartographic Journal, 37(1), 65-77, <https://doi.org/10.1179/caj.2000.37.1.65>.

Kwang, T.W. (2016). E-government global trends: integrated services, open data, e-participation and digital technologies, $<$ http://www.enterpriseinnovation.net/article/e-government-global-trendsintegrated-services-open-data-e-participation-and-digital>, consultado el 24 de junio de 2018.

Lee K.; Jeon J.; Lee W.; Jeong S.H. and Park S.W. (2003). QoS for Web Services: Requirements and Possible Approaches. W3C Working Group Note. World Wide Web Consortium (W3C), <http://www.w3c.or.kr/kr-office/TR/2003/wsqos>, consultado el 24 de junio de 2018.

Lee, J.G. and Kang M. (2015). "Geospatial Big Data: Challenges and Opportunities”, en Big Data Research (2):74-81.

López Pellicer, F.J. (2018). Comunicación personal.

Mani, A. and Nagarajan, A. (2002). Understanding quality of service for Web Services. Improving the performance of tour Web services. IBM. $<$ https://www.ibm.com/developerworks/library/ws-quality/index.html $>$, consultado el 24 de junio de 2018.

Menascé D.A. (2002). "QoS issues in Web services", en IEEE Internet Computing 6, 72-75.

Mesterton, N. and Kivekäs, R. (2018). "Towards Automating Spatial Data Quality Evaluation in the Finnish National Topographic Database", en International Workshop on Spatial Data Quality, Valletta (Malta), Eurogeographics, $<$ https://eurogeographics.org/wp-content/uploads/2018/06/14NTDB_show_Malta_2018_NM_RK.pdf>. 
Meyer, A. (2018). 5 blockchain trends influencing the future of social media marketing, <https://thenextweb.com/contributors/2018/04/19/5-blockchain-trendsinfluencing-future-social-media-marketing/>.

MIKE2 (2018). Method for an Integrated Knowledge Environment. $<$ http://mike2.openmethodology.org/>, consultado el 24 de junio de 2018.

Moellering, H. (ed.) (1987). A Draft Proposed Standard for Digital Cartographic Data. Report 8. Columbus, OH, National Committee for Digital Cartographic Data Standards.

Mueller, P.A. (1997). Modelado con objetos UML. Paris, France, Eyrolles.

NGAC (2016). Merging technologies and the geospatial landscape A Report of the National Geospatial Advisory Committee. NGAC Emerging Technologies Paper. Department of Interior.

NGAC (2017). Geospatial standards: a national asset. NGAC Emerging Technologies Paper. Department of Interior.

NIST (1994). Spatial data transfer standard (SDTS). Federal Information Processing Standards Publication 173-1. Gaithersburg, MD, USA, National Institute of Standards and Technology.

Oesterreich, T.D. and Teuteberg, F. (2016). "Understanding the implications of digitization and automation in the context of Industry 4.0: A triangulation approach and elements of a research agenda for the construction industry", en Computers in Industry, 83, 121-139.

OGC (2006). OpenGIS Web Map Server Implementation specification v 1.3.0. Wayland, MA, USA, Open Geospatial Consortium, $<$ http://www.opengeospatial.org/standards/wms $>$, consultado el 24 de junio de 2018.

OGC (2012). OGC Sensor Observation Service (SOS) Standard Version 2.0. Wayland, MA, USA, Open Geospatial Consortium.

OGC (2018). OGC Testbed-13: Aviation Abstract Quality Model Engineering Report. Open Geospatial Consortium. Wayland, MA, 01778, USA.

OXERA (2013). What is the economic impact of Geoservices?. Prepared for Google. London, United Kingdom, Oxera Consulting LLP.

Presutti, V.; d'Amato, C.; Gandon, F.; d'Aquin, M.; Staab, S. and Tordai, A. (eds.) (2014). "The Semantic Web: Trends and Challenges", en 11th International Conference, ESWC 2014, Anissaras, Crete, Greece.

Richardson, D. (2017). Five Tech Trends Driving New Geospatial Development. $<$ http://www.esri.com/esri-news/arcnews/summer17articles/five-tech-trendsdriving-new-geospatial-development>, consultado el 24 de junio de 2018.

Rodrigo, J.J. (2017). "Extracción de conocimiento mediante la aplicación de Inteligencia Artificial a la información espacial", en Jornadas de Ibéricas de Infraestructura

de

Datos

Espaciales, 
$<$ http://www.idee.es/resources/presentaciones/JIIDE17/Apresentacoes_JIIDE20 17_Extraccion_conocimiento_mediante_aplicacion_inteligencia_artificial_a_la_ informacion_espacial_Jose_Julio_Rodrigo_Bello.pdf $>$, consultado el 24 de junio de 2018.

Ruiz-Lendinez, J.J.; Ariza-López, F.J.; Ureña-Cámara, M.A. and Blázquez, L. (2011). "Digital map conflation: a review of the process and a proposal for classification", en International Journal of Geographical Information Science, 25(9), 1439-1466. DOI:10.1080/13658816.2010.519707.

Senaratne, H.; Mobasheri, A.; Ali, A.L.; Capineri, C. and Haklay, M. (2017). "A review of volunteered geographic information quality assessment methods", en International Journal of Geographical Information Science, 31(1), 139-167. DOI: 10.1080/13658816.2016.1189556.

Shukla, Y. (2014). AGI perspective on future trends in geospatial technologies. Association of geospatial industries, $<$ https://nrsc.gov.in/uim_2014_proceedings/papers_ppts/UIM2014_US2_AGI.p df $>$, consultado el 24 de junio de 2018.

Subbiah, G.; Alam, A.; Khan, L. and Thuraisingham, B. (2007). "Geospatial data qualities as web services performance metrics", en Proceedings of the 15th annual ACM international symposium on Advances in geographic information systems. New York, NY, USA, ACM: 66:1-66:4.

Ureña-Cámara, M.A.; Nogueras, F.J.; Lacasta, F.J. and Ariza-López, F.J. (2018). "A method for checking the quality of geographic metadata based on ISO 19157”, en International Journal of Geographic Information Science (pendiente de publicación).

Wikipedia (2018a). Vehículo autónomo, $<$ https://es.wikipedia.org/wiki/Veh\%C3\%ADculo_aut\%C3\%B3nomo>, consultado el 24 de junio de 2018.

Wikipedia (2018b). Trazabilidad, <https://es.wikipedia.org/wiki/Trazabilidad>, consultado el 24 de junio de 2018.

Wu, H.; Li, Z.; Zhang, H.; Yang, C. and Shen, S. (2011). "Monitoring and evaluating the quality of Web Map Service resources for optimizing map composition over the internet to support decision making", en Computers \&Geosciences 37, 85-94.

Xavier E.M.; Ariza-López F.J.; Chicaiza-Mora, E.G. and Buenaño, X. (2017). Modelo de Madurez-País relativo a la Calidad en IG. Proyecto Diagnóstico de la situación actual sobre las metodologías y procedimientos empleados para la evaluación de la calidad de la Información Geográfica (Proyectos Panamericanos de Asistencia Técnica -PAT- del Instituto Panamericano de Geografía e Historia, 2016). 
Xavier E.M.; Ariza-López F.J. and Ureña-Cámara, M.A. (2016). “A Survey of Measures and Methods for Matching Geospatial Vector Datasets", en $A C M$ Comput. Surv. 49(2), 1-34.

Xavier, E.M.A.; Ariza-López, F.J. and Ureña-Cámara, M.A. (2015a). "Web service for positional quality assessment: the WPS tier", en ISPRS Annals of the Photogrammetry, Remote Sensing and Spatial Information Sciences, II-3/W5. La Grande Motte, France, ISPRS, 257-262.

Xavier, E.M.A.; Ariza-López, F.J. and Ureña-Cámara, M.A. (2013). Geospatial Web Services Quality: an Overview and a Quality Model Proposal, Jaén, España, GIIC.

Xavier, E.M.A.; Ariza-López, F.J. and Ureña-Cámara, M.A. (2015b). "WPS for positional quality control applying the method proposed in UNE 148002", en VI Jornadas Ibéricas de Infraestructuras de Datos Espaciales, Sevilla, España, Grupo de Trabajo IDEE.

Yang, C.P.; Cao, Y.; Evans, J.; Kafatos, M. and Bambacus, M. (2006). "Spatial Web Portal for Building Spatial Data Infrastructure", en Journal of Geographic Information Sciences 12, 38-43.

Zeithaml, V.; Parasuraman, A. and Berry, L., (1990). Delivering Quality Service, New York, USA, The Free Press. 